\title{
Modélisation 3D simplifiée d'une plaque sur sol multicouche élastique
}

\section{F. CUIRA}

B. SIMON

TERRASOL

72, avenue Pasteur 93108 Montreuil Cedex f.cuira@terrasol.com
Cet article rend compte d'une méthode pour Y'analyse de l'interaction sol-plaque (radier, dallage industriel...). Le calcul consiste à établir un couplage entre les éléments finis de plaque et les formules de Boussinesq et conduit à des résultats voisins de ceux obtenus par traitement complet en éléments finis 3D. Ladite méthode a fait l'objet d'un programme informatique, TASPLAQ, qui permet de traiter le tassement et les sollicitations pour des plaques d'inertie et de forme variables, reposant sur un massif de couches horizontales, sous différents types de chargement. Le modèle a été perfectionné par plusieurs fonctionnalités complémentaires : prise en compte du décollement de la plaque, simulation de la plastification du sol en surface ainsi que la possibilité d'introduire différentes conditions de symétrie.

Mots-clés : modélisation 3D, interaction sol-structure, radier, dallage industriel, éléments finis de plaque, formules de Boussinesq, TASPLAQ.

\section{Simple 3D-modelling of a plate lying on a set of elastic layers}

This paper presents a calculation method for the analysis of soil-plate interaction (rafts, industrial pavements, etc.). This method consists in coupling plate finite elements with Boussinesq formulas, and leads to results that are very close to those reached with full 3D finite elements calculations. It was developed as a computer program called TASPLAQ, which enables to calculate settlements and bending moments for plates with various rigidities and shapes, and for various loading conditions. The plates are assumed to be laying on a set of horizontal elastics layers. The method was further improved with additional-options : possible separation of the plates from the soil, soil yielding under the plate edges and symmetries.

Key words : 3D-modelling, soil structure interaction, raft, industrial pavement, plate finite elements, Boussinesq formulas, TASPLAQ. 


\section{Introduction}

L'interaction sol-structure est un problème majeur qui a fait l'objet de nombreuses recherches et approches en France comme à l'étranger. Jusqu'à un passé récent, la méthode la plus courante consistait à considérer un appui continu élastique : les déplacements sont supposés proportionnels aux réactions et les calculs peuvent être menés soit à l'aide d'une formulation analytique, soit à l'aide de programmes informatiques. Cette approche constitue cependant une simplification radicale, car elle suppose qu'une charge ne provoque de tassements que dans sa zone d'application, ce qui n'est pas représentatif de la réalité. De plus, une telle approche ne permet pas de tenir compte de l'interaction entre deux structures voisines ou entre une structure et une charge extérieure s'appliquant directement au sol.

Une autre approche consiste à faire appel aux méthodes de calcul en éléments finis en trois dimensions : des méthodes qui, outre la multiplicité des paramètres, sont réputées complexes et lourdes, surtout pour des études préliminaires.

Cet article présente une approche intermédiaire permettant de répondre de manière satisfaisante à ce problème, dans un cadre dérivé de l'élasticité. Cette méthode repose sur les hypothèses suivantes (Fig. 1):

- le sol considéré est assimilé à un massif multicouche élastique, infini dans les directions horizontales $(\mathrm{Ox})$ et $(\mathrm{Oy})$. Chaque couche est horizontale et caractérisée par son module d'Young et son coefficient de Poisson ;

- le sol supporte une plaque, d'inertie et de géométrie variables, représentative d'un radier ou d'un dallage, et à laquelle on peut appliquer un chargement réparti ou concentré ;

- en plus de la pression exercée par la plaque, le sol peut être soumis à l'application d'une charge extérieure directe, représentative par exemple d'un remblai, pouvant interagir avec le système "sol-structure ).

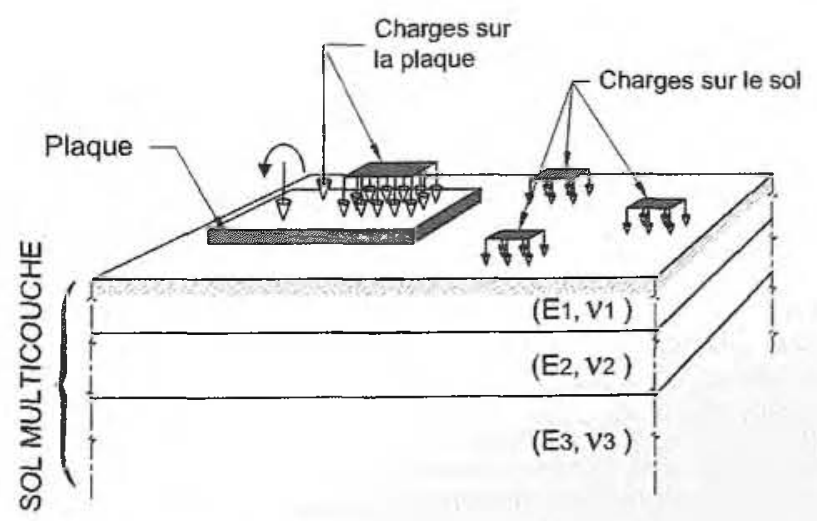

FIG. 1 Position du problème.

Presentation of the problem.

La méthode proposée permet de déterminer en tout point, le tassement, la réaction du sol ainsi que les sollicitations induites dans la plaque.

\section{Notations}

Dans toute la suite, les notations suivantes sont adoptées :

\section{NOTATIONS}

E : Module d'Young $(\mathrm{kPa})$

$v \quad$ Coefficient de Poisson

w : Flèche de la plaque, comptêe positivement vers le bas (m)

$\mathrm{M}_{\mathrm{x}} \quad$ : Moment de flexion autour de I'axe (-Oy) (kN.m/ml)

$\mathrm{M}_{\mathrm{y}} \quad$ : Moment de flexion autour de l'axe (Ox) (kN.m/ml)

$\mathrm{M}_{\mathrm{xy}}$ : Moment de torsion $(\mathrm{kN} . \mathrm{m} / \mathrm{ml})$

$\mathrm{T}_{\mathrm{x}}$; Effort tranchant suivant l'axe $(\mathrm{Ox})(\mathrm{kN} / \mathrm{ml})$

$\mathrm{T}_{\mathrm{y}}$ : Effort tranchant suivant l'axe $(\mathrm{Oy})(\mathrm{kN} / \mathrm{ml})$

n : Nombre total de nceuds activés du maillage éléments finis de la plaque

$\underline{K}^{e} \quad$ : Matrice de rigidité de la plaque $(3 n \times 3 n)$

$\underline{F}^{e}$ : Vecteur chargement équivalent résultant sur la plaque (3n)

$\underline{F}^{\text {ext }}$ : Vecteur chargement équivalent relatif aux efforts extérieurs (3n)

$\underline{B}^{s}$ : Vecteur chargement équivalent relatif à la réaction du sol (3n)

$\underline{a}^{e} \quad$ : Vecteur déplacement généralisé de la plaque (3n)

$\underline{\mathbf{T}}_{\text {inf }}$ : Matrice de souplesse (ou d'influence) du sol $(n \times n)$

S : Vecteur tassement résultant du sol

$\underline{P}^{s}$ : Vecteur réaction du sol (n)

$\underline{\mathbf{s}}^{\text {ext }}$ : Vecteur tassement représentant la part du tassement résultant des charges extérieures s'appliquant directement au sol (n)

$\mathrm{T}^{s} \quad$ : Matrice de passage permettant d'exprimer $\underline{\mathbf{R}}^{s}$ en fonction de $\underline{\mathbf{P}}^{s}(3 \mathrm{n} \times \mathrm{n})$

G : Matrice de passage permettant d'exprimer

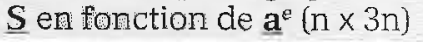

\section{5 \\ Référence \\ à quelques méthodes existantes}

Plusieurs méthodes de calcul des radiers ou dallages ont été proposées pour offrir des approches intermédiaires entre la méthode " aux coefficients de réaction» et les éléments finis 3D permettant d'aborder ces problèmes d'interaction sol-structure ; toutes reposant sur des hypothèses plus ou moins simplificatrices. 
P. Vezole (1986) a abordé ce problème dans un cadre bidimensionnel (problème plan ou à symétrie de révoIution), en proposant une approche consistant à discrétiser la structure étudiée (radier, semelle ou dallage...) en plusieurs tronçons et à combiner cette discrétisation avec les formules usuelles relatives au calcul des tassements d'un massif élastique semi-infini. L'auteur a appliqué cette approche à quelques cas courants pour mettre en lumière les simplifications implicitement introduites par la méthode "aux coefficients de réaction ».

Dans la même optique, M. Cassan et al. (1991) ont mis au point une approche simplifiée pour l'étude de l'interaction sol-radier, dont le principe est proche de celui de la méthode objet de cet article. Leur appro che est basée, pour l'équilibre du radier, sur l'intégration numérique de l'équation de Lagrange. Pour le sol support, elle consiste à faire appel à la distribution de contraintes de Boussinesq, supposée applicable même en présence d'un substratum. Il a été vérifié, sur des cas simples, que cette approche conduit à des résultats très voisins de ceux obtenus par traitement complet en éléments finis.

Pour le cas des dallages, le DTU 13.3 fournit une méthode simplifiée, basée sur la combinaison des formules de Boussinesq et de celles relatives à une plaque infinie sur ressorts juxtaposés. Cette méthode permet de calculer les déformations et les sollicitations dans le corps du dallage dues à différents types de chargement.

Ces trois approches ont deux facteurs en commun qu'il convient de souligner : l'utilisation des formules de Boussinesq pour le calcul des tassements et l'adoption du modèle simplifié des plaques (ou poutres) minces pour calculer l'équilibre de la fondation.

On peut également citer la méthode couramment utilisée pour le dimensionnement des chaussées, quj consiste à assimiler l'ensemble ( chaussée + sol ) à un multicouche élastique, en résolvant rigoureusement le problème de Burmister (1943). Cette méthode puissante, ne permet cependant pas de prendre en compte les efforts de bord puisque l'élément de fondation est supposé infini en plan. Également, sa résolution numérique qui n'est actuellement réalisée qu'en coordonnées cylindriques, ne s'applique qu'à des charges circulaires; le cas des charges rectangulaires est traité en considérant un ou plusieurs disques d'emprise équivalente.

\section{Notions théoriques préliminaires}

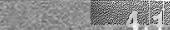 \\ Modélisation d'une plaque en flexion pure}

La plaque est supposée localement homogène isotrope, de comportement élastique linéaire. On se limite au cas des plaques travaillant en flexion pure, et dont l'épaisseur est faible devant les autres dimensions, ce qui permet d'adopter la théorie classique des plaques minces et plus exactement « le modèle discret de Kirchhoff ), où seules les déformations dues à la flexion sont prises en compte : la contribution des déformations de cisaillement est donc négligée.

Dans le cadre de ce modèle, deux systèmes d'équations régissent le comportement et l'équilibre de la plaque :
- équations d'équilibre $\left\{\begin{array}{l}\mathrm{T}_{\mathrm{x}}+\left(\frac{\partial \mathrm{M}_{\mathrm{x}}}{\partial \mathrm{x}}+\frac{\partial \mathrm{M}_{\mathrm{xy}}}{\partial \mathrm{y}}\right)=0 \\ \mathrm{~T}_{\mathrm{y}}+\left(\frac{\partial \mathrm{M}_{\mathrm{y}}}{\partial \mathrm{y}}+\frac{\partial \mathrm{M}_{\mathrm{xy}}}{\partial \mathrm{x}}\right)=0 \\ \mathrm{q}+\left(\frac{\partial \mathrm{T}_{\mathrm{x}}}{\partial \mathrm{x}}+\frac{\partial \mathrm{T}_{\mathrm{y}}}{\partial \mathrm{y}}\right)=0\end{array}\right.$

- loi de comportement $\left\{\begin{array}{l}M_{x}=\frac{E I}{\left(1-v^{2}\right)}\left(\frac{\partial^{2} w}{\partial x^{2}}+v \frac{\partial^{2} w}{\partial y^{2}}\right) \\ M_{y}=\frac{E I}{\left(1-v^{2}\right)}\left(\frac{\partial^{2} W}{\partial y^{2}}+v \frac{\partial^{2} w}{\partial x^{2}}\right) \\ M_{x y}=\frac{2 E I}{\left(1-v^{2}\right)}\left(\frac{\partial^{2} w}{\partial x y}\right)\end{array}\right.$

où $q$ désigne la densité de charge appliquée localement à la plaque; EI, le produit d'inertie (on rappelle que $\mathrm{EI}=\frac{\mathrm{Eh}^{3}}{12}$, E étant le module d'Young de la plaque et h son épaisseur) ; $v$, le coefficient de Poisson.

La combinaison des équations (1) et (2) conduit à l'équation (3), dite de Lagrange :

$$
\frac{\partial^{4} W}{\partial x^{4}}+2 \frac{\partial^{4} W}{\partial x^{2} \partial y^{2}}+\frac{\partial^{4} W}{\partial y^{4}}=q \frac{\left(1-v^{2}\right)}{E I}
$$

La résolution de cette équation peut être menée à l'aide d'une formulation en éléments finis. On a choisi un modèle de type “ déplacements » avec des éléments rectangulaires à quatre nœuds et douze degrés de liberté (Zienkiewicz et Taylor, 1991) : chaque nœud possède ainsi trois degrés de liberté, une translation et deux rotations, sachant que ces rotations ne sont autres que les premières dérivées partielles de la flèche (approximation de Kirchhoff).

Ce modèle, simple à mettre en œuvre, converge par ailleurs de manière sûre et rapide.

\section{2}

\section{Modélisation du sol : formules de Boussinesq}

\section{A propos des formules de Boussinesq}

Le sol est assimilé à un massif multicouche élastique. Certes, tous les géotechniciens savent bien que le sol n'est pas un matériau élastique, mais Terzaghi, lui-même, admettait que lorsque les contraintes sont inférieures au tiers des valeurs limites, on peut considérer avec une approximation suffisante que le sol a un comportement élastique.

Le comportement élastique du sol étant admis, on se propose d'utiliser les formules de Boussinesq pour le calcul des tassements en surface. Ces formules qui ont été établies pour le cas d'un massif homogène semiinfini, peuvent être étendues au cas d'un multicouche en admettant que les distributions de contraintes sont identiques dans les deux cas. Burland et al. (1977) ont discuté de manière approfondie les conditions de cette simplification; ils ont conclu que celle-ci était effectivement acceptable dans la majorité des situations couramment rencontrées par l'ingénieur. Une exception 
notable est le cas d'une couche raide surmontant des couches plus déformables.

\section{Calcul des tassements}

Le calcul des tassements est fondé principalement sur la formule de Steinbrenner qui découle des formules de Boussinesq (Terzaghi, 1943). Cette formule permet d'exprimer le tassement, dû̀ à une couche située entre les profondeurs D1 et D2 (Fig. 2), sous le coin d'une charge rectangulaire uniforme $q$ :

$\rho_{\mathrm{D} 1-\mathrm{D} 2}=\frac{\mathrm{qB}}{\mathrm{E}}\left(\left(1-v^{2}\right)\left(\mathrm{F}_{1}\left(\mathrm{~d}_{2}\right)-\mathrm{F}_{1}\left(\mathrm{~d}_{1}\right)\right)+\left(1-v-2 v^{2}\right)\left(\mathrm{F}_{2}\left(\mathrm{~d}_{2}\right)-\mathrm{F}_{2}\left(\mathrm{~d}_{1}\right)\right)\right)(4)$

avec :

$$
\left\{\begin{array}{l}
F_{1}(d)=\frac{1}{\pi}\left(\ln \left(\frac{\left(1+\sqrt{1^{2}+1}\right) \sqrt{1^{2}+d^{2}}}{1\left(1+\sqrt{1+d^{2}+l^{2}}\right)}\right)+\ln \left(\frac{\left(1+\sqrt{1^{2}+1}\right) \sqrt{1+d^{2}}}{\left(1+\sqrt{1+d^{2}+l^{2}}\right)}\right)\right) \\
F_{2}(d)=\frac{d}{2 \pi} \arctan \left(\frac{1}{d \sqrt{1+d^{2}+l^{2}}}\right) \quad d=\frac{D}{B} \text { et } l=\frac{L}{B}
\end{array}\right.
$$

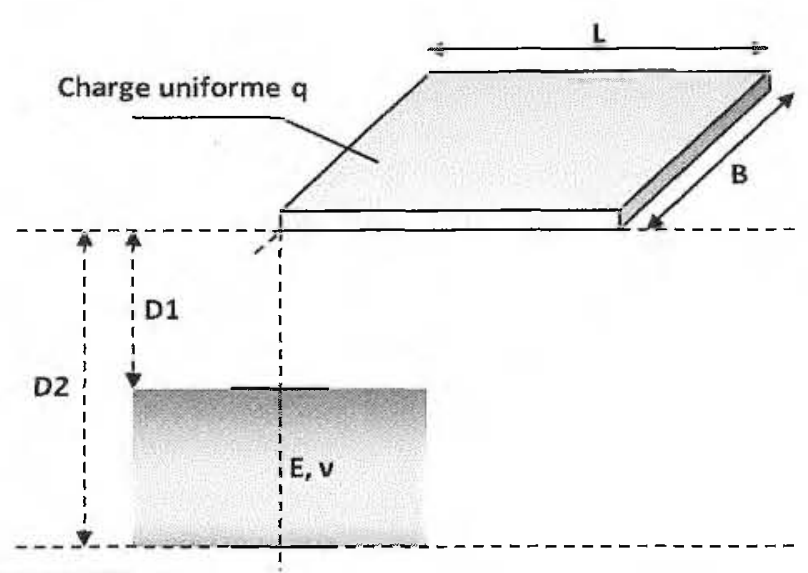

FIG. 2 Application de la formule de Steinbrenner. Application of the Steinbrenner's formula.

Ainsi, par addition des tassements produits dans chaque couche, l'application de cette formule, combinée avec la méthode de superposition algébrique, permet d'évaluer le tassement induit par une ou plusieurs charges rectangulaires, en tout point de surface d'un multicouche élastique.

\section{5}

\section{Principes théoriques de la méthode}

La plaque est discrétisée en éléments finis rectangulaires. Si l'on admet que la réaction du sol est uniformément répartie autour de chaque nœud, on peut alors exprimer les tassements aux nouds en fonction de la réaction du sol à l'aide d'une " matrice de souplesse » dont le calcul peut être conduit sur la base des caractéristiques de chaque couche à l'aide des formules de Boussinesq. La formulation finale du problème est obtenue ensuite en écrivant l'égalité, en chaque nœud, entre le tassement du sol et le déplacement vertical de la plaque.

\section{Modélisation de la plaque}

La discrétisation de la plaque est réalisée à l'aide d'un maillage dont le ( pas ) peut varier dans les deux directions (Fig. 3). Les éléments utilisés sont les éléments rectangulaires de Kirchhoff présentés précédemment. Chaque élément est caractérisé par son module d'Young $E$, son épaisseur h et son coefficient de Poisson v. Il convient de noter que le choix du coefficient de Poisson influe de façon notable sur les moments calculés dans la plaque. En particulier, si on choisit $v=0$, alors le moment dans chaque direction est proportionnel à la courbure associée, ce qui n'est pas le cas si $v \neq 0$. Dans le cas d'une plaque en béton, il est d'usage de prendre $v=0,2$ si le béton est non armé et $v=0$ dans le cas d'un béton armé.

Le chargement extérieur appliqué à la plaque est introduit à l'aide de charges réparties associées à chaque élément, et de charges ponctuelles (effort vertical + deux moments fléchissants) associées à chaque nœud du maillage.

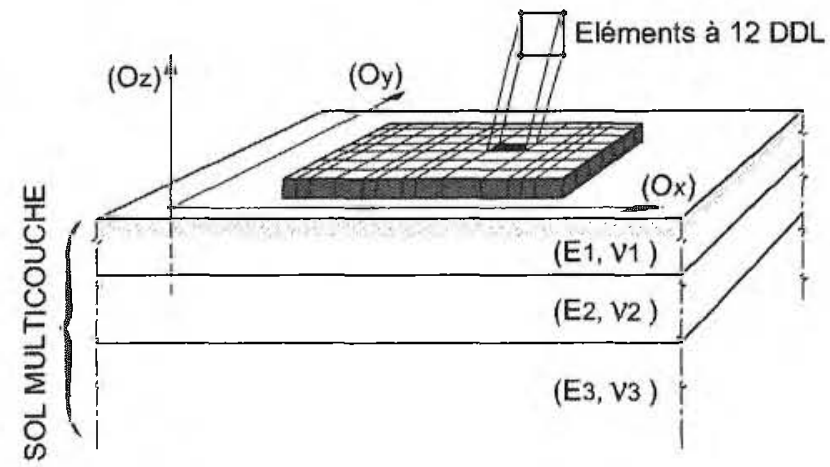

FIG. 3 Modélisation de la plaque en éléments finis.

Modelling of the plate by the finite element method.

Par ailleurs, une technique de « désactivation des éléments » permet de modéliser une plaque de forme polygonale, l'existence de trous intérieurs, ou encore le cas de plusieurs plaques désolidarisées (Fig. 4).

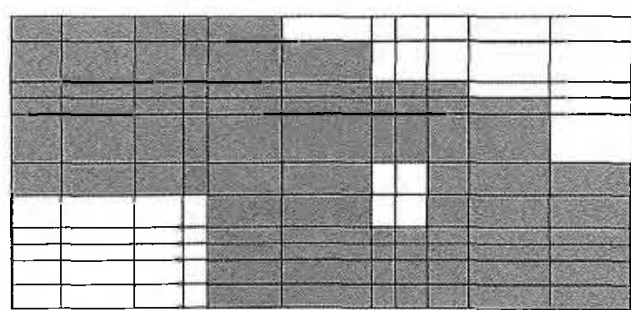

Modélisation d'une plaque de forme polygonale

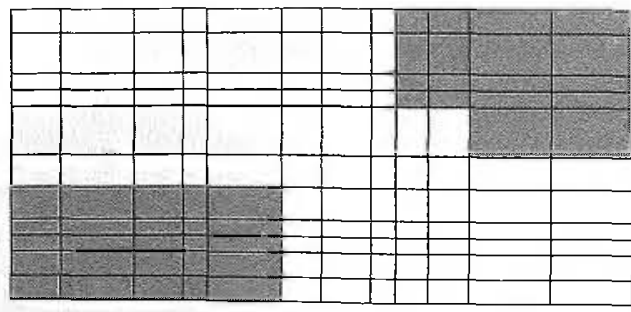

Modélisation de plusieurs plaques indépendantes

Eléments désactivés

Eléments activés

FIG. 4

Modélisation d'une forme polygonale, modélisation de plusieurs plaques.

Modelling of a polygonal shape, modelling of several plates. 
Pour tout ce qui suit, on désigne par « $n$ ) le nombre total des nœuds (activés) du maillage. A l'aide de ce maillage, l'équation d'équilibre de la plaque se traduit par un système linéaire (6) :

$$
\underline{\underline{\mathbf{K}}}^{e} \cdot \mathbf{a}^{e}=\mathbb{F}^{e}
$$

où $\underline{K}^{e}$ est la matrice de rigidité de la plaque $(3 n \times 3 n)$, constituée par assemblage des matrices de rigidité associées à chaque élément du maillage; $\mathbf{F}^{e}$, le vecteur chargement équivalent de la plaque (3n), constitué par assemblage des « vecteurs chargement » élémentaires; $\mathbf{a}^{\mathrm{e}}$, le vecteur déplacement équivalent de la plaque (3n), constitué par le déplacement et les rotations en chaque noud.

En examinant les efforts équilibrant la plaque (Fig. 5), on peut décomposer le vecteur chargement en deux parties : un vecteur chargement relatif aux efforts extérieurs $\mathbb{F}_{-}^{c x t}$, et un vecteur chargement relatif à la réaction du sol $\mathbf{R}^{s}$ :

$$
\underline{\underline{\mathbf{K}}}^{e} \cdot \mathbf{a}^{e}=\mathbf{E}^{e x t}-\mathbf{R}^{s}
$$

Le premier vecteur est calculé directement à l'aide des charges appliquées sur la plaque. Le deuxième fait intervenir la rigidité du sol.

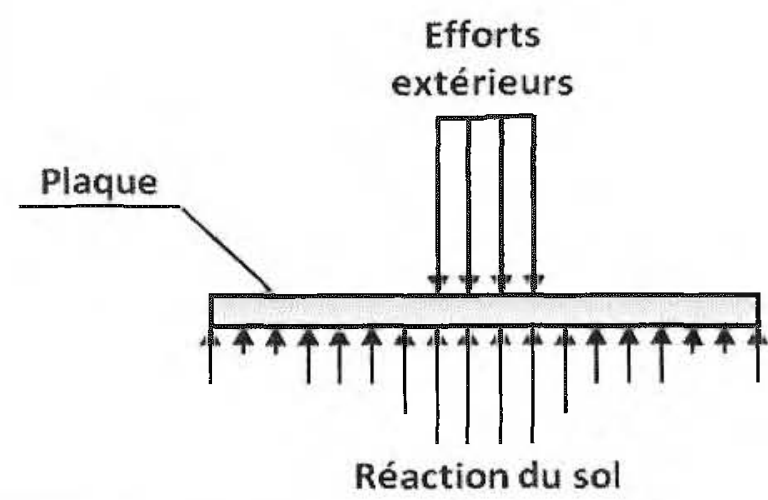

FIG. 5 Équilibre de la plaque. Equilibrium of the plate.

\section{Modélisation de l'interaction sol-plaque}

On se place dans l'hypothèse d'un contact sans frottement: seules les réactions normales à la plaque sont prises en compte. On néglige ainsi les efforts de cisaillement à l'interface sol-plaque.

Par le principe d'action-réaction, la réaction du sol n'est autre que la pression exercée par la plaque sur celui-ci. L'idée de base consiste à supposer que cette pression est uniforme autour de chaque nœud : plus exactement, elle est uniforme sur un rectangle délimité par les centres des éléments connectés à ce noud. Il faut bien sûr tenir compte du cas particulier d'un nœud situé sur le bord, d'un nœud situé en un coin sortant ou rentrant. Pour une plaque de forme polygonale, la distribution des pressions d'interaction a la forme schématisée sur la figure 6 .

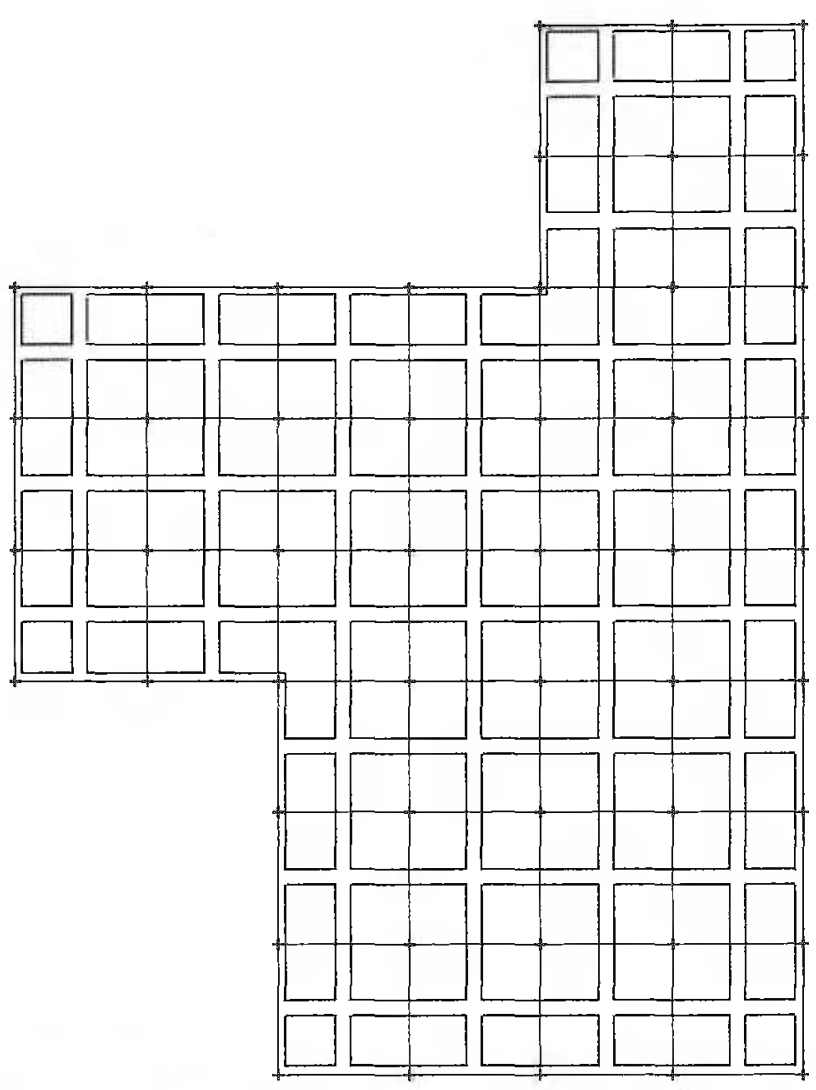

FIG.6 Distribution de la réaction du sol sous les éléments de plaque.

Distribution of soil reactions under plate elements.

\section{Modélisation du sol support}

Le sol est assimilé à un massif multicouche élastique. Chaque couche est caractérisée par son module d'Young, son épaisseur et son coefficient de Poisson. L'influence de ce dernier est généralement faible, et il est d'usage de prendre une valeur comprise entre 0,25 et 0,35 . Le module d'Young est souvent estimé en référence aux résultats des essais pressiométriques, en adoptant une valeur égale à $\mathrm{k} \frac{\mathrm{E}_{\mathrm{M}}}{\alpha}, \mathrm{E}_{\mathrm{M}}$ étant le le module pressiométrique ; $\alpha$, le coefficient rhéologique, et $\mathrm{k}$ un facteur majorant, pouvant varier entre 1 et 4 , et qui dépend à la fois du type de sol, de la dimension de la fondation ainsi que du niveau de déformation atteint. O. Combarieu (2006) a discuté de manière approfondie du choix de la valeur de $\mathrm{k}$; il a notamment montré qu'un calcul élastique mené, comme il est souvent constaté, en adoptant le choix de la valeur $\mathrm{k}=1$, peut conduire à des tassements pessimistes.

A l'aide du maillage associé à la plaque, on définit une ( matrice de souplesse ) du sol notée $\underline{\underline{T}}_{\mathrm{inf}}(\mathrm{n} \times \mathrm{n})$, qui permet de relier linéairement les pressions exercées sur le sol aux tassements induits par celles-ci (8); de telle sorte que le coefficient ( $\left.\alpha_{i j}\right)$ est calculé en examinant le tassement induit au noeud ( $\mathrm{i}$ ) par une pression unitaire appliquée autour du nœud « $\mathrm{j}$ ). Le calcul est conduit à l'aide de la méthode présentée en 4.2 . 


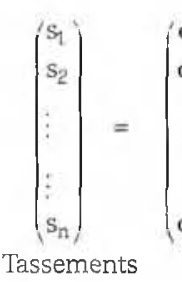
$\underline{S}$
Matrice de souplesse

$\underline{T}_{\text {ini }}$

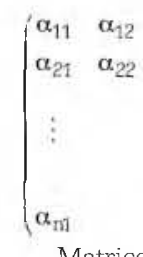

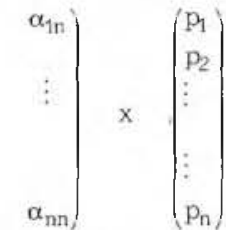

Pressions dinteraction

$\underline{P}^{s}$
Bien noter que cette matrice n'est pas symétrique, autrement dit, on a dans le cas général : $\alpha \neq \alpha$

Par ailleurs, le tassement du sol en surface résulte à la fois de la pression exercée par la plaque et des charges extérieures s'appliquant directement au sol (Fig. 1). On écrit donc :

$$
\underline{\mathbf{S}}=\underline{\underline{\mathbf{T}}}_{\mathrm{inf}} \mathbf{P}^{\mathrm{s}}+\underline{\mathbf{S}}^{\text {ext }}, \quad \mathbf{P}^{\mathrm{s}}=\left(\underline{\underline{T}}_{\mathrm{inf}}\right)^{-1} \cdot\left(\underline{\mathbf{S}}-\underline{\mathbf{S}}^{\mathrm{ext}}\right)
$$

où $\mathbf{S}^{\text {ext }}$ désigne la part du tassement générée par ces charges extérieures.

\section{5}

\section{Formulation du problème et résultats}

On reprend à présent l'équation d'équilibre de la plaque : $\underline{\mathbf{K}}^{\mathrm{e}} \cdot \underline{\mathbf{a}}^{\mathrm{e}}=\mathbf{F}^{\text {ext }}-\underline{\mathbf{R}}^{\mathrm{s}}$. Le terme $\mathbf{R}^{s}$ s'exprime linéai- $^{\prime}$ rement en fonction de la réaction du sol $\mathbf{P}^{s}$. Ensuite, à l'aide de la matrice de souplesse, on exprime la réaction du sol en fonction du tassement (9). Enfin, la formulation finale du problème s'obtient en écrivant l'égalité, en chaque nœud, entre le tassement du sol et le déplacement vertical de la plaque

$$
\left(\underline{\underline{\mathbf{K}}}^{\mathrm{e}}+\underline{\underline{\mathbf{T}}}^{\mathrm{s}} \cdot(\underline{\underline{\mathbf{T}}} \underline{\underline{n f}})^{-1} \cdot \underline{\underline{\mathbf{G}}}\right) \cdot \underline{\mathbf{a}}^{\mathrm{e}}=\mathrm{F}^{\mathrm{ext}}+\underline{\underline{\mathbf{T}}}^{\mathrm{s}} \cdot\left(\underline{\underline{\mathbf{T}}}_{\mathrm{in}}\right)^{-1} \cdot \underline{\underline{\mathbf{s}}}^{\mathrm{ext}}
$$

où $\mathbf{T}^{s}$ est la matrice de passage qui permet d'exprimer $\underline{\mathbf{R}}^{s}$ en fonction de $\mathbf{P}^{s} ; \mathbf{G}$, la matrice qui permet de passer de $\mathbf{S}$ à $\mathbf{a}^{e}$ grâce à l'égalité en chaque noud entre le tassement et le déplacement vertical de la plaque.

La résolution de ce système permet d'évaluer le vecteur déplacement généralisé $\mathbf{a}^{\circ}$, et donc les variables nodales associées à chaque élément du maillage. Cela permet de calculer :

- la flèche $w$ de la plaque en tout point de chaque élément ;

- la réaction du sol en tout point à l'aide de (9) ;

- le tassement du sol en tout point (y compris au droit des parties désactivées) ;

- les moments fléchissants dans la plaque, que l'on peut évaluer à partir de la flèche de la plaque définie dans chaque élément à l'aide de (2). Notons que les moments sont calculés uniquement à l'intérieur de chaque élément (en quatre points dits « points d'intégration ») du fait du caractère « non conforme » des éléments utilisés, qui n'assure pas la continuité des efforts lors du passage d'un élément à un autre.

\section{5}

\section{Traitement de certains cas particuliers}

\section{Décollement de la plaque}

Le calcul précédent est valable dans l'hypothèse où la plaque demeure en contact avec le sol. Le sol ne pouvant générer des efforts de traction, l'obtention de réactions négatives nécessite de considérer les nœuds correspondants comme « décollés ». Ceci est réalisé à l'aide d'un calcul itératif automatique moyennant un critère de décollement : un seuil de traction au-delà duquel on considère qu'il n'y a plus de liaison entre la plaque et le sol en surface. Ainsi, pour les nœuds considérés comme « décollés »:

- le déplacement de la plaque n'est plus égal au tassement du sol ;

- la réaction du sol au droit de ces nouds est nulle.

Un retraitement adapté de la matrice de souplesse (8) permet de tenir compte de ces nouvelles conditions.

\section{Limitation de la réaction du sol}

Dans certains calculs élastiques, on aboutit souvent à un pic de réaction au bord de la plaque (exemple d'une semelle chargée uniformément). Dans la discrétisation de plaque adoptée, ce pic de réaction est d'autant plus important que la largeur des éléments placés au bord est réduite. Pour un sol réel, il y a limitation de ce pic par déformation plastique.

Cette limitation peut être contournée (mais seulement partiellement) en contrôlant la taille des éléments placés au bord pour créer les conditions d'une diffusion de ce pic de réaction. Une autre manière plus élaborée consiste à simuler la plastification du sol à l'interface sol-plaque en menant un calcul itératif automatique, tel qu'au droit des nœuds considérés comme « plastifiés », la réaction du sol est imposée égale à un seuil de plastification prédéfini, tandis que pour les zones non plastifiées, l'expression élastique des tassements est conservée. On s'assure enfin que la répartition des réactions obtenues respecte bien l'équilibre statique de la plaque.

\section{Prise en compte d'une contrainte initiale dans le sol}

Il s'agit de traiter le cas où une contrainte initiale (ou une contrainte ( avant travaux $)$ ) existe dans le sol, par exemple le cas d'un radier fondé au fond d'une excavation. L'hypothèse adoptée consiste à ne transmettre au sol que la fraction de la charge qui excède le poids des terres initial. Ceci peut être pris en compte en adaptant la relation (9):

$$
\mathbf{S}=\underline{\underline{\mathbf{T}}}_{\text {inf }}\left(\mathbf{P}^{\mathrm{s}}-\sigma_{v}^{0}\right)+\mathbf{S}^{\mathrm{ext}}
$$

où $\mathrm{o}_{v}^{0}$ est le vecteur des contraintes verticales initiales.

\section{6}

\section{Mise en ceuvre de la méthode}

La méthode présentée a fait l'objet d'un programme informatique, nommé TASPLAQ, qui a la capacité de traiter:

- le cas d'une plaque de géométrie et d'inertie variables, soumise à un chargement qui peut être réparti (charges verticales) ou ponctuel (efforts verticaux et moments) ;

- le cas d'un sol support défini comme un massif multicouche élastique, qui peut être soumis, en plus de la pression exercée par la plaque, à des charges extérieures directes ; 
- la gestion automatique du décollement de la plaque ainsi que de la limitation locale des pressions d'interaction.

Le programme permet également d'introduire des ressorts élastiques sous la plaque, avec des raideurs en rotation ou en translation, ponctuelles ou réparties.

Par ailleurs, afin de permettre le traitement de certains cas particuliers, le programme a été adapté pour tenir compte de différents cas de symétrie : symétrie par rapport à un ou deux plans, symétrie par rapport à un axe, ou encore le cas des déformations planes. Dans chaque cas, la condition de symétrie est supposée valable pour la plaque, pour le sol, ainsi que pour le chargement.

La symétrie par rapport à un axe correspond au cas d'une plaque circulaire soumise à un chargement à symétrie de révolution. Le traitement d'un tel cas nécessite d'adopter des modèles particuliers : pour la plaque, on adopte le modèle de Kirchholf avec des éléments annulaires à deux nouds el quatre degrés de Iiberté ; pour le sol, une intégration numérique des formules de Boussinesq est réalisée pour le calcul du tassement d'une charge annulaire uniformément répartie.

Le cas des déformations planes correspond à la situation où la flexion de la plaque peut être négligée dans une direction. Dans ce cas, on utilise pour la fondation des éléments finis de poutre, tandis que pour le sol, il suffit d'adopter les formules de Boussinesq utilisées pour le cas d'un plan semi infini (problème de Flamant).

\section{7}

\section{Quelques exemples}

Lobjet de cette partie est d'illustrer la mise en pratique de la méthode TASPLAQ à travers quatre exemples retenus volontairement schématiques et simples: le cas d'une dalle sous chargement ponctuel, celui d'un dallage avec diflérents systèmes de joints, celui d'une semelle chargée localement, ou encore le cas de l'interaction entre un radier et un remblai.

\section{1. $1.9-19$}

\section{Exemple $1:$ dalle rectangulaire sous charges concentrées voisines}

Une dalle rectangulaire de $20 \mathrm{~m}$ de côté est soumise à l'application de deux charges voisines de $5(x) \mathrm{kN}$ chacune. Les données du problème sont détaillées sur la figure 7. La dalle a été discrétisée à l'aide du maillage représenté sur la fïgure 8 , qui a été raftiné autour des deux points d'application des charges. Ces deux charges ont été introduites dans TASPI AQ comme des charges ponctuelles verticales. On ne tient pas compte du poids propre de la dalle dans cet exemple.

Le calcul permet de déterminer en tout point, le tassement, la réaction du sol, ainsi que les moments fléchissants. Ces résultats peuvent être présentés graphiquement d'une manière surfacique en 3D ou en taisant des coupes suivant des axes particuliers.

Les figures 9 et 10 illustrent les résultats obtenus au droit de l'axe (AX) représenté sur la fïgure 8. La courbe de tassements révèle deux " pics ) proches des points

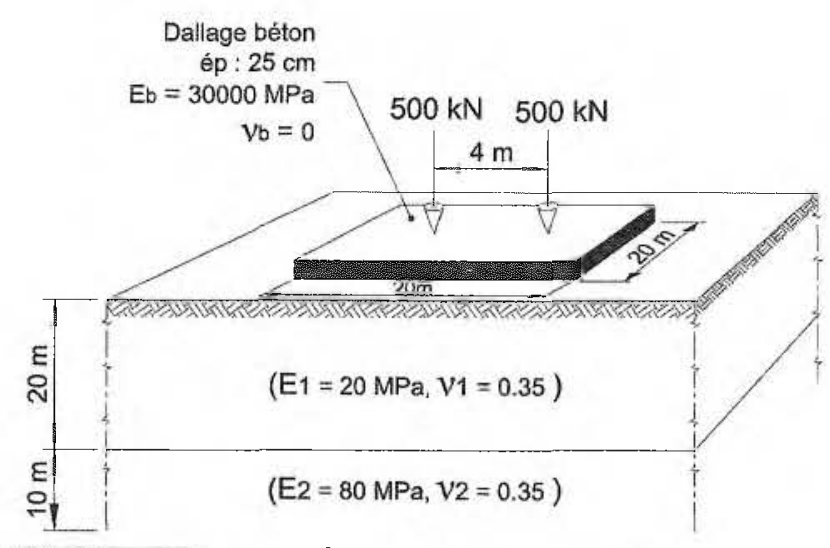

FIG. 7 Exemple 1: données du problème. General data for the example 1.

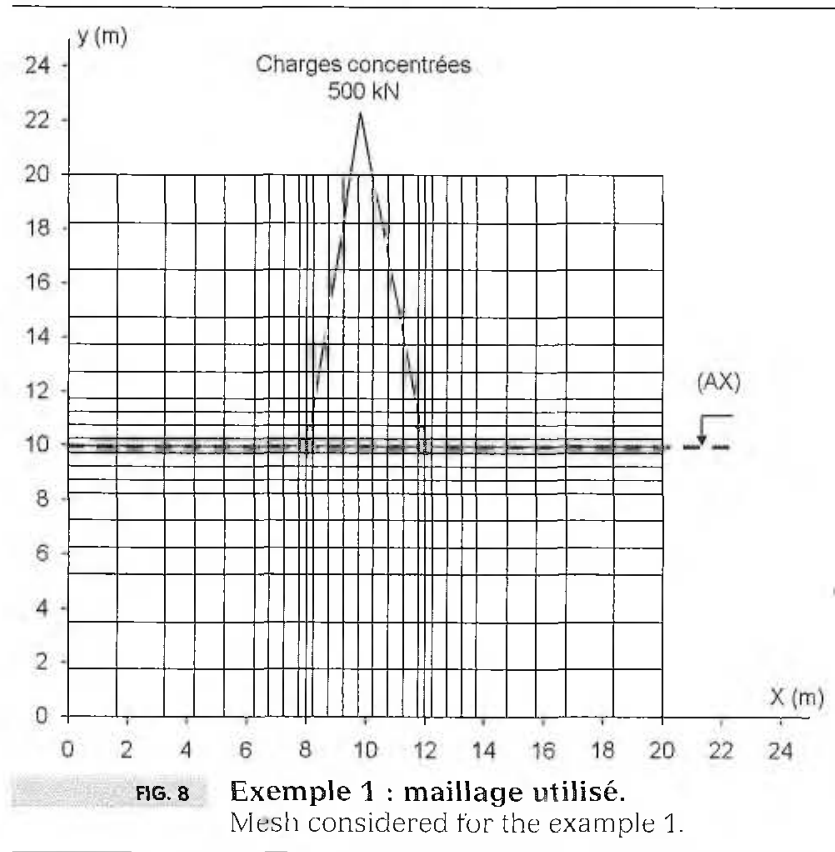

d'application des charges. On retrouve le caractère ( en escalier ) de la réaction du sol (uniforme autour de chaque nœud). La réaction maximale est de $45 \mathrm{kPa}$ pour un tassement de l'ordre de 6,6 $\mathrm{mm}$.

Au niveau des sollicitations, il est à noter que le cas d'un chargement ponctuel demeure purement théorique; il conduit en toute rigueur à des valeurs infinies de moment aux points d'application des charges. Dans le cas présent, le maillage de la plaque introduit une diffusion implicite des deux efforts qui explique les valeurs obtenues.

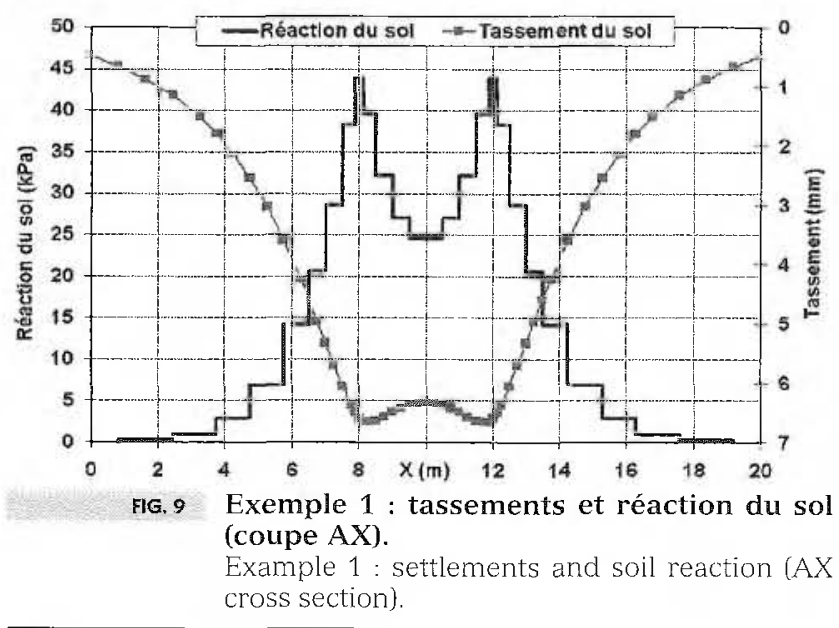




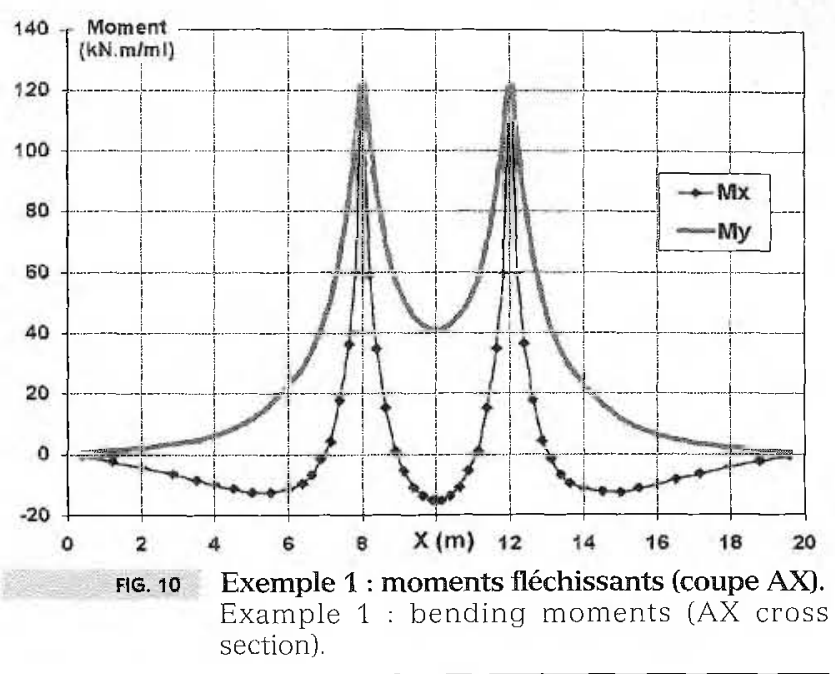

\section{2.}

\section{Exemple 2 : dallage rectangulaire avec différents systèmes de joints}

Un dallage rectangulaire de $16 \mathrm{~m} \times 10 \mathrm{~m}$ est soumis, en plus de son poids propre, à une charge de $50 \mathrm{kPa}$ répartie sur $10 \mathrm{~m} \times 5 \mathrm{~m}$ en son centre. L'objet de cet exemple est d'illustrer l'aptitude de la méthode à traiter également le cas des dallages avec différents systèmes de joints. Trois situations ont été étudiées : le cas d'un dallage continu (sans joints), le cas des joints partiels et le cas des joints francs. Les figures 11 et 12 détaillent les données du problème.

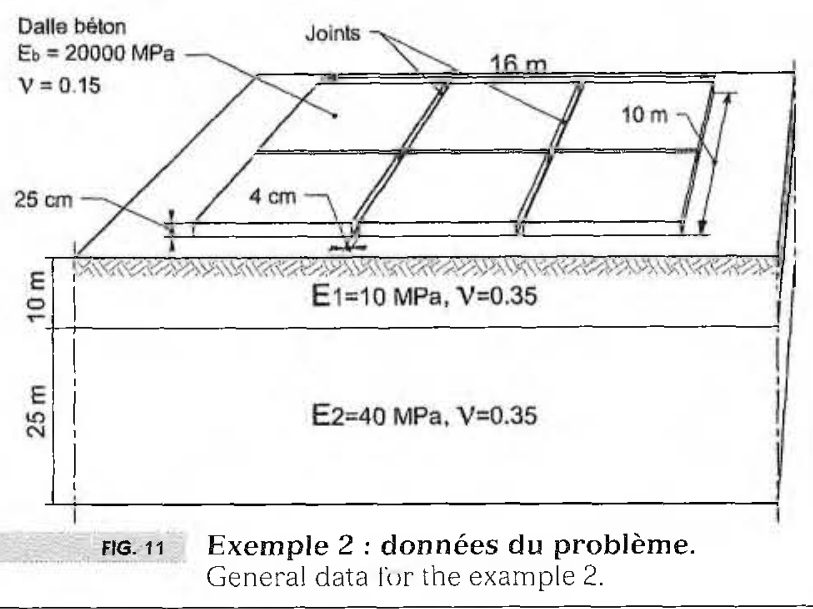

Le dallage a été discrétisé selon le maillage représenté sur la figure 13. Des éléments intermédiaires y ont été introduits permettant de simuler la présence éventuelle des joints : dans le cas d'un dallage continu, on affecte à ces éléments les mêmes caractéristiques mécaniques que celles du corps de dallage; dans le cas d'un dallage avec joints partiels, on a supposé que le dallage a été scié sur $4 / 5$ de son épaisseur (Fig. 12), ce qui revient à affecter à ces éléments une épaisseur réduite égale à $5 \mathrm{~cm}$; enfin, dans le cas d'un dallage avec joints francs, ces éléments sont tout simplement désactivés.

Pour le chargement, en plus de la charge centrale de $50 \mathrm{kPa}$, on tient compte du poids propre du dallage modélisé comme une charge de $6,25 \mathrm{kPa}$ répartie sur toute la surface de la plaque. Enfin, un critère de décollement de $0 \mathrm{kPa}$ a été considéré : aucune traction n'est donc autorisée à l'interface sol-plaque.

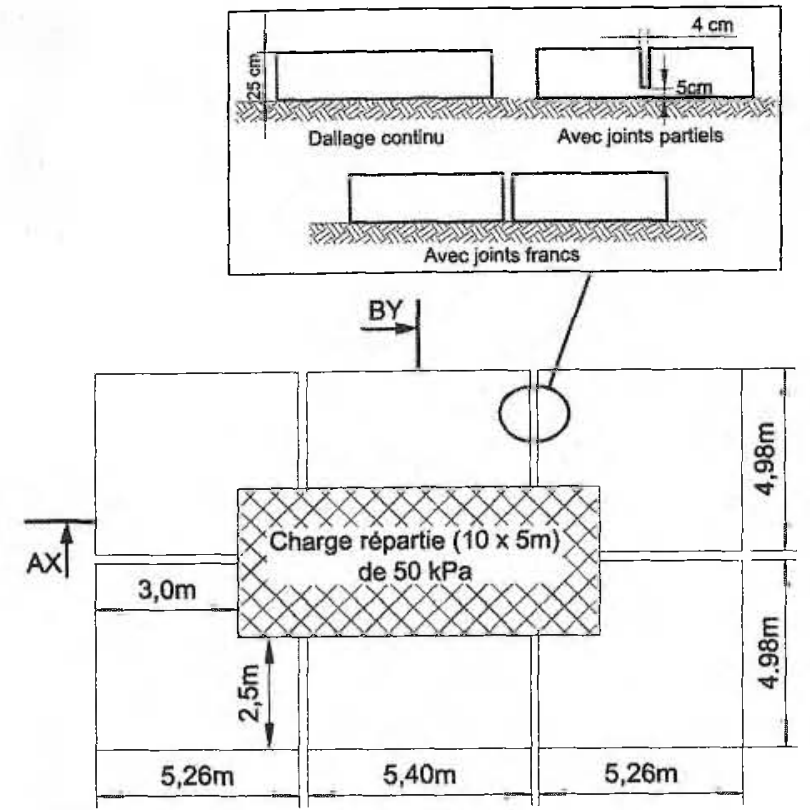

FIG. 12 Zone de chargement et disposition des joints éventuels.

Loading area and location of joints.

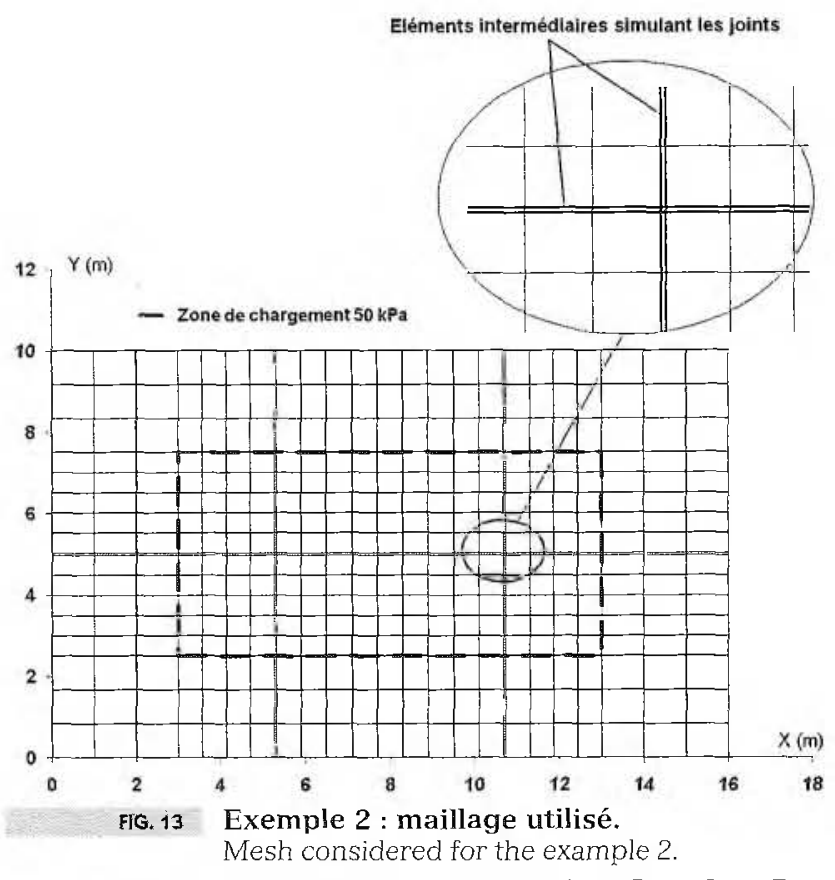

Les figures 14 et 15 présentent les résultats obtenus en termes de tassements et de moments au droit des coupes AX et BY identifiées sur la figure 12. Ces résultats montrent que la présence des joints modifie notablement l'amplitude et l'allure des moments dans les deux directions. Les sollicitations dans le corps du dallage sont ainsi atténuées et les tassements sont légèrement augmentés en transformant progressivement le dallage continu initial en six panneaux désolidarisés demeurant en interaction réciproque. Notons que dans le cas ultime des joints francs, l'interaction entre les six panneaux du dallage illustrée par le modèle TASPLAQ, ne peut pas être retrouvée dans un calcul où le sol est assimilé à des ressorts juxtaposés. 

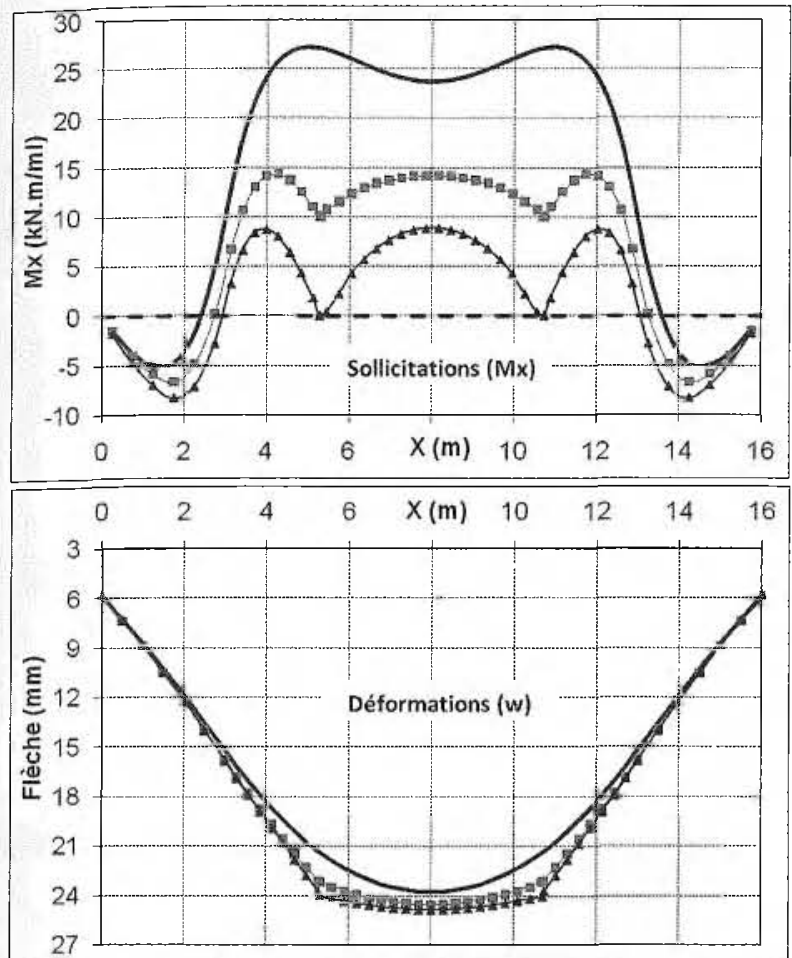

-Dallage continu sans joints -Dallage avec joints partiels $\rightarrow$ Dallage avec joints francs

FIG. 14 Comparaison des trois situations (coupe AX).

Comparison of the three situations (AX cross section).
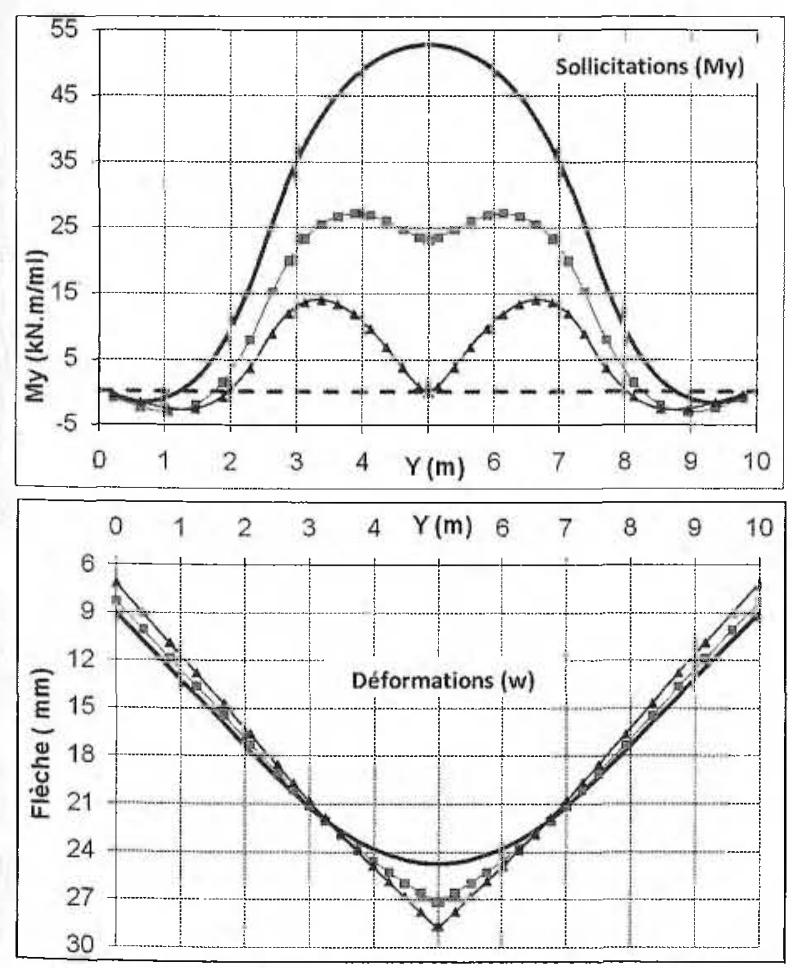

-Dallage continu sans joints

$\rightarrow$ Dallage avec joints francs

FlG. 15 Comparaison des trois situations (coupe BY).

Comparison of the three situations (BY cross section).

\section{Exemple 3 : semelle de roulement chargée localement}

Une semelle de roulement de $30 \mathrm{~m}$ de longueur et de $6 \mathrm{~m}$ de largeur, est soumise à une charge de $150 \mathrm{kPa}$ appliquée sur sa poutre centrale sur un linéaire de $10,5 \mathrm{~m}$. Les données du problème sont détaillées sur la figure 16. La semelle a été discrétisée selon le maillage de la figure 17, où sont également représentés les deux axes de symétries du problème (AX) et (BY) situés respectivement à $\mathrm{y}=3 \mathrm{~m}$ et $\mathrm{x}=15 \mathrm{~m}$.

Dans cet exemple, le poids propre de la semelle a volontairement été négligé et un critère de décollement de $0 \mathrm{kPa}$ a été considéré : aucune traction n'est donc autorisée à l'interface sol-plaque.

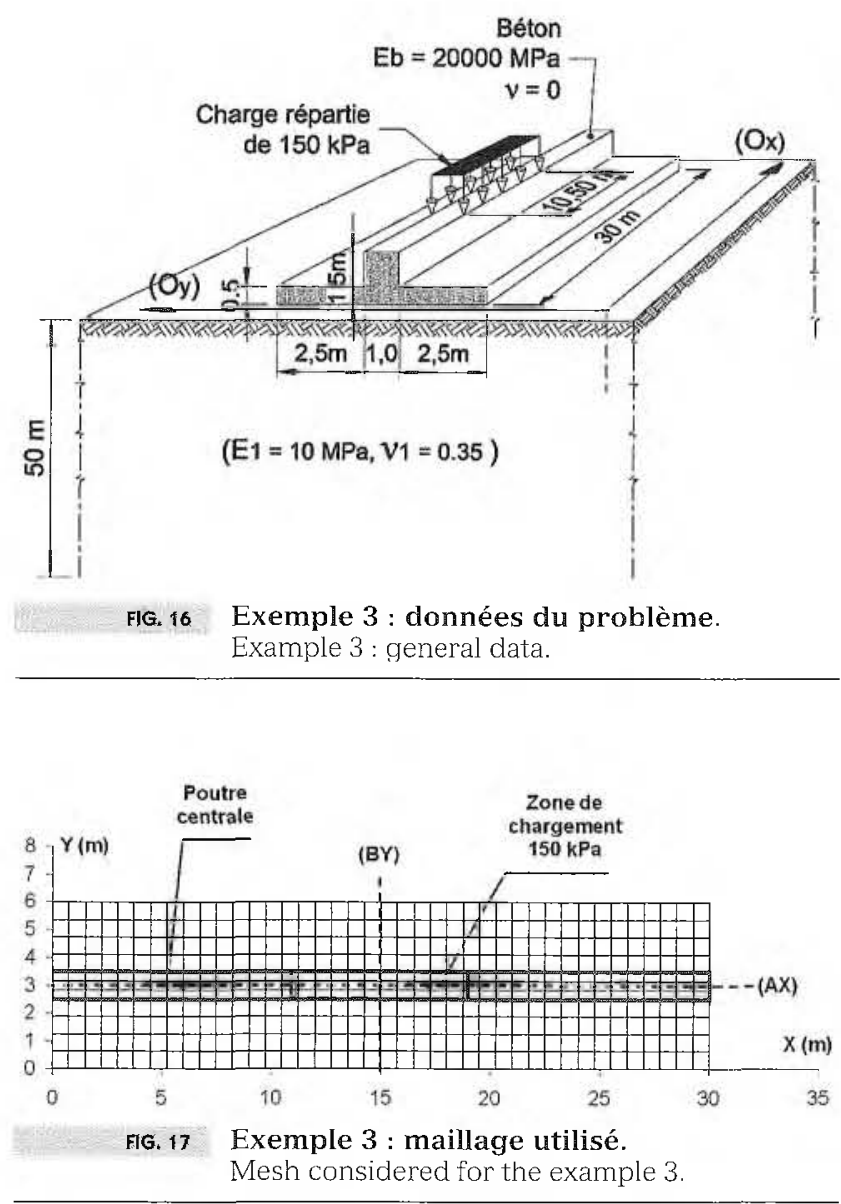

Les figures 18 et 19 présentent les résultats obtenus, au droit des axes (AX) et (BY), en termes de flèche, de tassements ainsi que de réaction du sol. Ces résultats montrent qu'avec le critère de décollement considéré, une zone de décollement est observée aux deux extrémités de la semelle : ceci résulte en partie du fait que le poids propre de la semelle ait été négligé. L'exemple retenu volontairement schématique illustre bien l'aptitude du modèle à gérer les décollements. La figure 19 illustre également « l’effet de bord » qui se caractérise par une augmentation du rapport « contraintes/déformations " au voisinage des bords de la semelle. On peut enfin remarquer que la semelle, par sa rigidité, diffuse la charge qui lui est appliquée : la réaction du sol au centre de la semelle n'excède pas $14 \mathrm{kPa}$. 


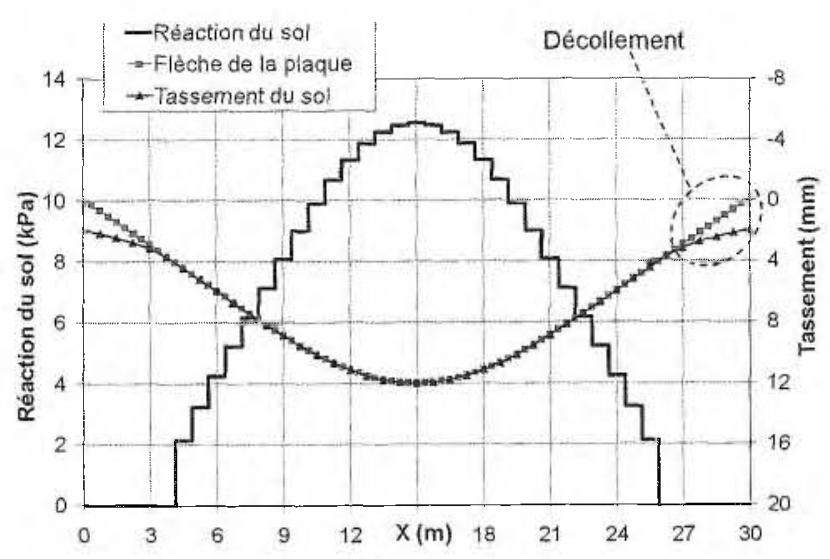

FIG. 18 Exemple 3 : flèche de la plaque, tassements et réaction du sol (coupe $\mathrm{AX}$ ).

Example 3: plate deflection, settlements and soil reaction (cross section $\mathrm{AX}$ )

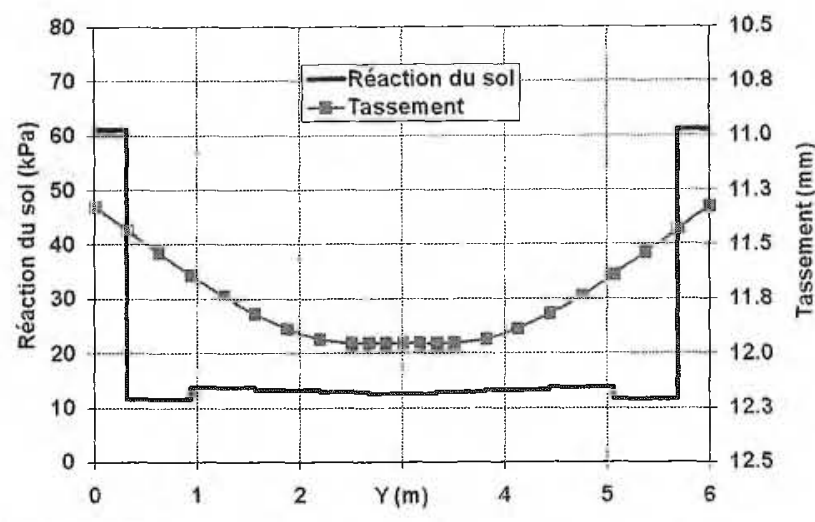

FIG. 19 Exemple 3 : tassements et réaction du sol (coupe BY).

Example 3 : settlements and soil reaction [cross section $\mathrm{BY}$.

La figure 20 présente les résultats obtenus en termes de moments fléchissants $\mathrm{Mx}$ et $\mathrm{My}$. Ces résultats montrent que le moment $\mathrm{Mx}$ est repris principalement par l'âme de la semelle.

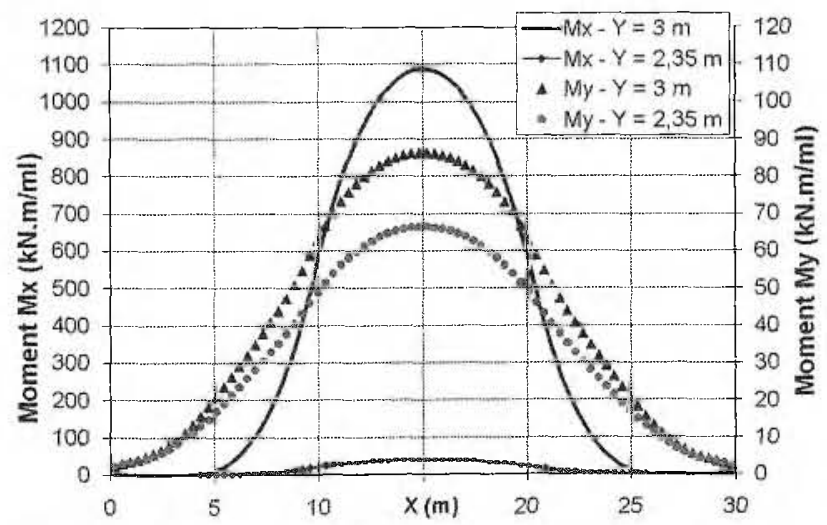

FlG. 20 Exemple 3 : moments fléchissants $\mathrm{Mx}$ et My.

Example 3 : bending moments $\mathrm{Mx}$ and $\mathrm{My}$

\section{Exemple 4 : radier au voisinage d'un remblai}

Un radier en forme de ( $L$ ) est soumis, en plus de l'action de son poids propre, à l'application de trois charges concentrées de $300 \mathrm{kN}$ chacune. Ce radier est situé au voisinage d'un remblai de $2 \mathrm{~m}$ de hauteur, comme le schématise la figure 21.

Le radier a été discrétisé selon le maillage représenté sur la figure 22, dont une zone est désactivée pour suivre la géométrie de la plaque. Dans ce maillage, chacune des charges concentrées a une surface d'impact de $1 \mathrm{~m} \times 1 \mathrm{~m}$. Le poids propre du radier est modélisé par une charge répartie de $12,5 \mathrm{kPa}$ appliquée à toute la surface de la plaque. Enfin, le remblai est pris en compte comme une charge extérieure de $40 \mathrm{kPa}$, s'exerçant directement sur le sol au droit de la zone désactivée. Le remblai est ainsi implicitement supposé appliqué en même temps que les charges sur le radier.

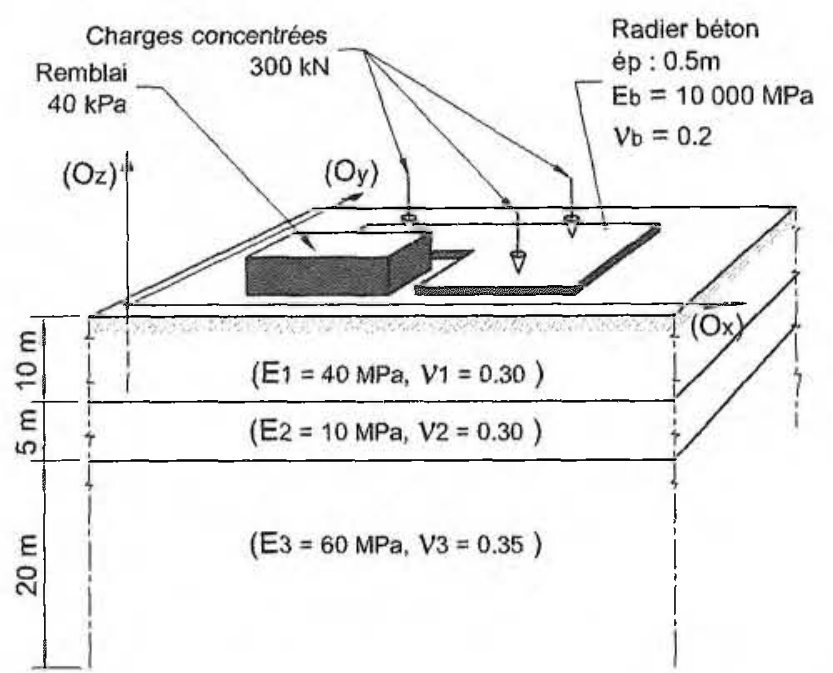

FGG. 21 Exemple 4 : données du problème. Example 4 : general data.

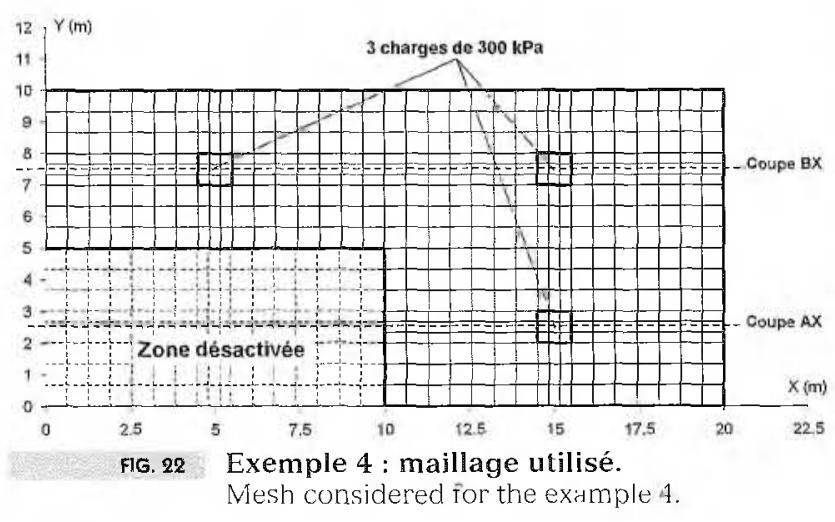

Les résultats peuvent être comparés à ceux obtenus en ignorant la présence du remblai. La figure 23 présente les tassements au droit des coupes (AX) et (BY) identifiées sur la figure 22. Ces résultats illustrent bien l'influence du remblai qui modifie notablement l'allure et l'amplitude des tassements sous le radier. L'interaction radier - remblai mise en évidence ne peut pas être retrouvée par un "calcul sur ressorts » où les charges n'ont une influence qu'au droit des zones où elles sont appliquées. 


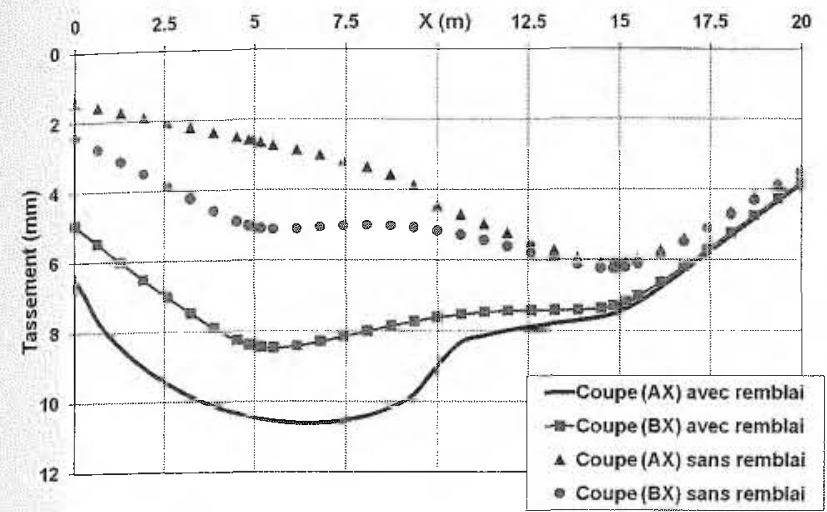

F1G. 23 Exemple 4 : influence du remblai sur les tassements en surface.

Example 4 : interaction with an embankment settlements
TABLEAUI Comparaison des tassements (mm). Comparison of settlements (mm)

\begin{tabular}{lccc} 
& TASPLAQ & PLAXIS 3D & Rapport $\rho$ \\
\hline Exemple 1 & 6,6 & 6,6 & $\mathbf{1 , 0 0}$ \\
Exemple 2 ${ }^{(1)}$ & 24,8 & 25,8 & $\mathbf{0 , 9 6}$ \\
Exemple 3 & 12,0 & 12,1 & $\mathbf{0 , 9 9}$ \\
Exemple 4 & 10,7 & 9,9 & $\mathbf{1 , 0 8}$ \\
\hline
\end{tabular}

TABIEAU II Comparaison des moments maximaux $\mathrm{Mx}(\mathrm{kN} . \mathrm{m} / \mathrm{ml})$.

Comparison of bending moments $\mathrm{Mx}(\mathrm{kN} \cdot \mathrm{m} / \mathrm{ml})$.

\begin{tabular}{lccc} 
& TASPLAQ & PLAXIS 3D & Rapport $\rho$ \\
\hline Exemple 1 & 108 & 110 & $\mathbf{0 , 9 8}$ \\
Exemple 2 $^{(1)}$ & 28,4 & 28,7 & $\mathbf{0 , 9 9}$ \\
Exemple 3 $^{11}$ & 1090 & 1070 & $\mathbf{1 , 0 2}$ \\
Exemple 4 & 60 & 61 & $\mathbf{0 , 9 8}$ \\
\hline
\end{tabular}

TABLEAU III Comparaison des moments maximaux $\mathrm{My}(\mathrm{kN} . \mathrm{m} / \mathrm{ml})$.

Comparison of bending moments My (kN.m/ml).

\begin{tabular}{lccc} 
& TASPLAQ & PLAXIS 3D & Rapport $\rho$ \\
\hline Exemple 1 & 122 & 118 & $\mathbf{1}, \mathbf{0 3}$ \\
Exemple $2^{(1)}$ & 53 & 53 & $\mathbf{1 , 0 0}$ \\
Exemple 3 & 86 & 87 & $\mathbf{0 , 9 9}$ \\
Exemple 4 & 56 & 57 & $\mathbf{0 , 9 8}$ \\
\hline
\end{tabular}

Là méthode présentée a lait l'objet d'une étude de validation qui s'est appuyée sur des tests de compa raison avec d'autres méthodes. Une première étape a consisté à valider le modèle de plaque utilisé, à savoir celui de Kirchhofî à quatre nceuds et douze degrés de liberté, en vérifianl que dans le cas d'une plaque sur appuis élastiques par exemple, on aboutit bien à des résultats comparables à ceux issus des autres modèles de calcul de structure couramment utilisés (analytiques et éléments fïnis). Une deuxième étape a consisté à valider la méthode dans le cas général d'une plaque sur massif multicouche élastique. Il a pu être établi que, dans le domaine de validité des hypothèses adoptées, TASPLAQ conduit à des résultats voisins de ceux issus d'un traitement complet en éléments finis en trois dimensions, avec des écarts n'excédant pas $10 \%$ en général, ce qui est acceptable el convient aux applications pratiques de l'ingénieur.

De manière générale, les écarts constatés sont essentiellement attribués aux limites résultant de la généralisation des formules de Boussinesq au cas d'un multicouche : ces limites, rappelons-le, concernent le cas où les rigidités des couches sunt contrastées; elles ne sont toutefois significatives que lorsqu'une couche raide est rencontrée immédiatement en surface, ce qui n'est pas le cas le plus courant. On constate néanmoins que dans ces cas spécifiques, la rigidité propre à l'élément de structure (plaque du radier ou dallage) atténue notablement les écarts en tassement qui serajent obtenus sans cet élément.

A titre de comparaison, les exemples présentés précédemment ont été modélisés en éléments finis en trois dimensions à l'aide du logiciel PLAXIS 3D (pris comme modèle de référence). Les tableaux I à III présentent les résultats obtenus en termes de tassements el de moments lléchissants :

\section{avec $\rho=\frac{\text { Valeur TASPLAQ }}{\text { Valeur PLAXIS3D }}$}

La comparaison des tassements (tableau I) conduit à des valeurs de $\rho$ variant entre 0,96 et 1,08 . On constate que TASPLAQ sous-estime légèrement les tassements dans la situation où les rigidités des couches croissent avec la profondeur $(\rho<1$, exemples 1 à 3). En revanche, une surestimation des tassements est observée lorsque les rigidités des couches supérieures décroissent avec la profondeur $(\rho>1$, exemple 4).

Par contraste, les écarts associés aux moments (tableaux II et III) sont plus faibles avec des valeurs de $\rho$ variant entre 0,98 et 1,03 . Les figures 24 et 25 confirment et clarifient cette tendance : on observe en effet que les écarts obtenus en tassements n'induisent que des différences faibles au niveau des sollicitations calculées dans la plaque.

Par ailleurs, la méthode TASPLAQ présente des avantages décisifs par rapport aux éléments finis 3D. Tout d'abord, "l'emprise » du modèle est limitée à celle des éléments de fondation; sa construction s'en trouve donc notablement simplifiée puisqu'il n'est pas nécessaire de mailler le sol en profondeur et autour de la plaque. Ceci facilite le raffinement du maillage, là où cela est nécessaire (charges, joints...), sans peser sur la taille globale du modèle. Enfin, l'expérience montre que les temps de préparation et de calcul sont réduits, notamment pour les problèmes

\footnotetext{
(1) Comparaison établie pour le cas d'un dallage continu
} 
impliquant des phénomènes de décollement puisque le modèle demeure appuyé sur une approche initiale élastique.
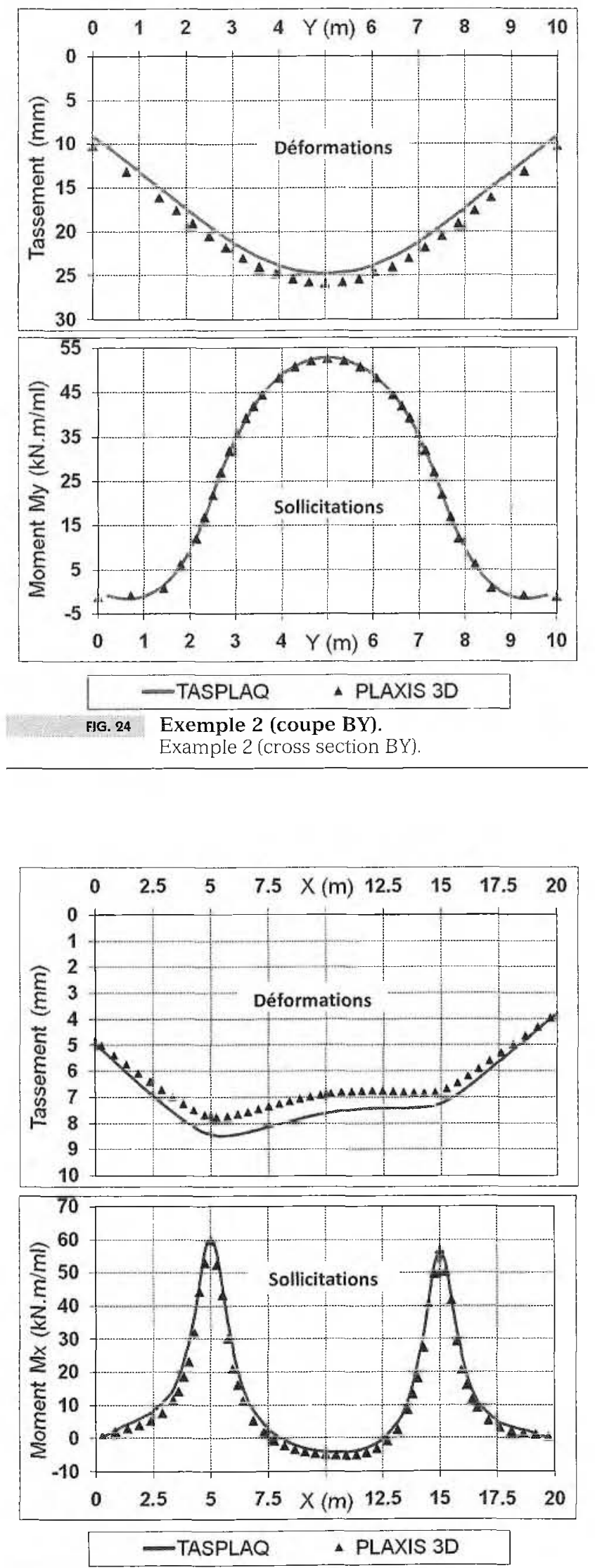

FIG. 25 Exemple 4 (coupe BX.

Example 4 (cross section BX).

\section{Comparaison avec les règles de calcul du DTU 13.3}

L'objet de cette partie est de comparer les résultats issus de TASPLAQ à ceux obtenus par l'application des règles de calcul de la norme dallages (DTU 13.3). Pour cela, on se place dans les conditions de l'exemple 1, avec deux cas de chargement (Fig. 26) : le cas d'une charge isolée de $500 \mathrm{kN}$ au centre du dallage (point O) et le cas de deux charges de $500 \mathrm{kN}$ chacune, espacées de $4 \mathrm{~m}$ (aux points A et B)
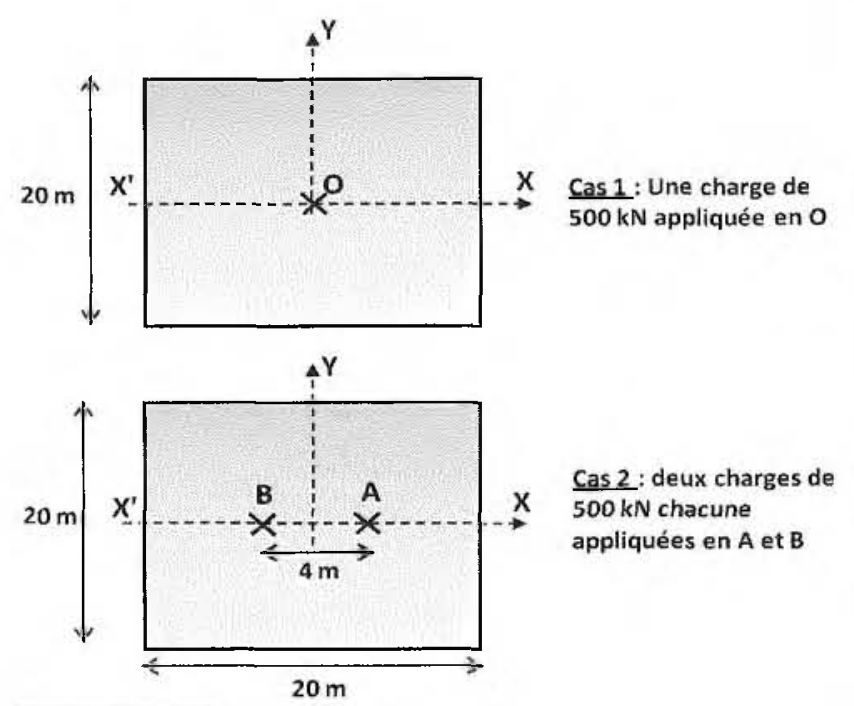

Cas 2: deux charges de $500 \mathrm{kN}$ chacune appliquées en A et B

FIG. 26 Cas étudiés : localisation des points de chargement.

Studied cases : location of loading points.

\section{Application des règles de calcul du DTU 13.3}

Ces règles, explicitées dans l'annexe C du DTU 13.3, couvrent la détermination des sollicitations subies par le dallage d'origine mécanique (liées aux charges appliquées) ou d'ordre rhéologique (liées au comportement du béton : retrait, gradient thermique, dessiccation...). Seules les sollicitations d'origine mécanique sont traitées par la méthode TASPLAQ, les autres sollicitations devant ensuite leur être combinées au titre du projet complet de l'ouvrage. On se limite donc dans cette partie à la comparaison des déformations et sollicitations d'origine mécaniques, dues aux « charges d'exploitations $»$, obtenues par les deux méthodes.

Dans le cas d'une ou plusieurs charges concentrées en partie courante, la méthode décrite dans le DTU 13.3 repose sur deux notions

- le diamètre équivalent, noté $\mathrm{D}_{\text {eq, }}$ égal à celui d'une zone de support circulaire qui, soumise à l'application directe d'une charge uniformément répartie, subit en son centre un tassement identique à celui provoqué sur le dallage par une charge concentrée d'intensité égale à la résultante de cette charge répartie ;

- le module conventionnel de réaction du support, noté $\mathrm{K}_{\mathrm{eq}}$ égal au rapport entre la pression uniformément répartie sur la zone de support de diamètre $\mathrm{D}_{\mathrm{eq}}$, et le tassement en son centre. 
Dans l'exemple étudié d'un sol bicouche, le calcul de ces deux paramètres se fait de manière itérative. On obtient les valeurs suivantes:

$$
\mathrm{D}_{\text {eq }}=5,42 \mathrm{~m}, \mathrm{~K}_{\text {eq }}=4700 \mathrm{kPa} / \mathrm{m}
$$

Le diamètre équivalent obtenu est associé à chaque charge concentrée appliquée en partie courante du dallage. Les déformations induites par ces charges sont calculées par la formule suivante:

$$
\mathrm{w}=\sum_{1}^{\mathrm{n}} \mathrm{w}_{\mathrm{i}} \cdot \mathrm{C}_{\mathrm{P}\left(\mathrm{x}_{\mathrm{i}}, 0\right)}
$$

où w est le tassement provoqué par la charge concentrée d'indice i au droit de cette charge; $\mathrm{C}_{\mathrm{p}}\left(\mathrm{x}_{\mathrm{i}} \mathrm{O}\right)$, le coefficient de propagation du tassement qu'on calcule à partir des coefficients d'influence relatifs à un support homogène et les caractéristiques mécaniques de chaque couche $; x_{i}$, la distance de la charge d'indice i au point de calcul.

Pour les sollicitations, le DTU 13.3 suggère une méthode de calcul s'appuyant sur la courbure du support et fournissant les moments conventionnels dus à différents types de chargement.

\section{Cas d'une charge isolée}

Sur la figure 27, on a représenté les tássements

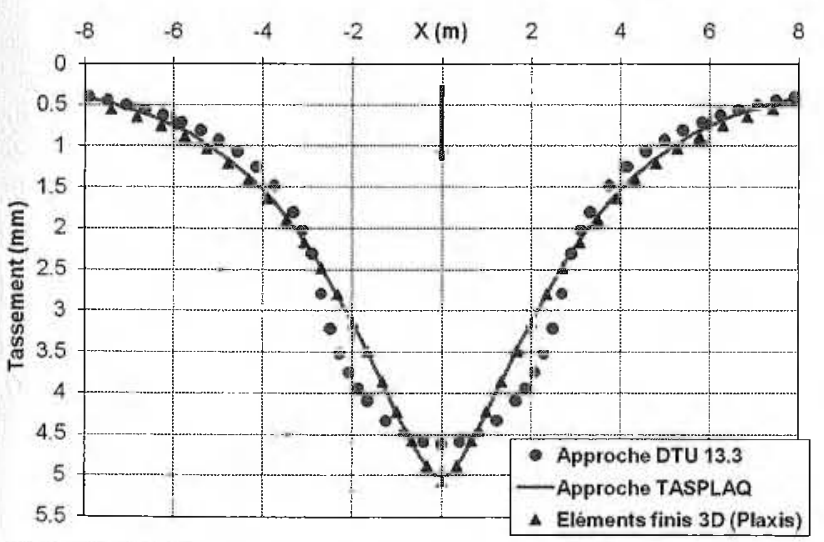

FIG. 27 Cas d'une charge isolée : tassements (axe $X^{\prime} X$ ). Case of a poinl load: settlements ( $\mathrm{X}^{\prime} \mathrm{X}$ axisl.

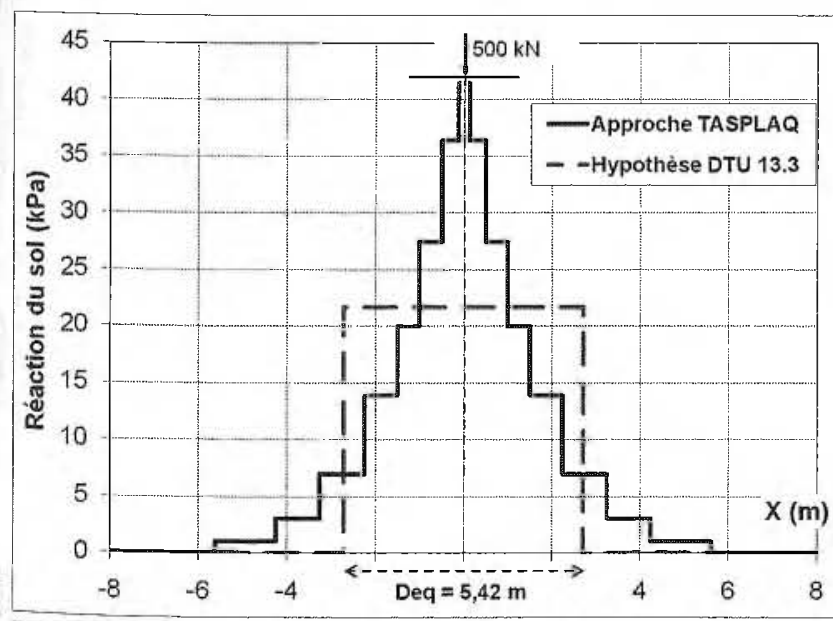

FIG. 28 Cas d'une charge isolée : réaction du sol (axe X'X). Case of a point load: soil reaction ( $\mathrm{X}^{\prime} \mathrm{X}$ axis). issus de TASPLAQ ainsi que ceux obtenus par l'approche du DTU 13.3. Les tassements obtenus par un traitement complet en éléments finis 3D sont également figurés. Ces résultats montrent que la méthode du DTU 13.3 fournit une déformée approchée du dallage avec des tassements légèrement sous-estimés mais du même ordre de grandeur que ceux fournis par TASPLAQ ou PLAXIS 3D.

Au niveau des réactions, la figure 28 montre que l'hypothèse du DTU 13.3, qui considère que la réaction du sol est uniforme sur un disque centré sur la charge, n'est qu'une approximation par rapport au résultat issu d'une approche plus élaborée.

Quant aux sollicitations, on rappelle que pour un calcul de plaque, le cas d'une charge ponctuelle demeure purement théorique ; il conduit en toute rigueur à des valeurs infinies de moment au point d'application de la charge. Une manière usuelle pour simuler ce cas consiste à considérer une diffusion de la charge ponctuelle sur le plan neutre de la plaque, avec un angle de diffusion de 45 degrés, comme le montre la figure 29. Ainsi, sous TASPLAQ ou PLAXIS, la charge concentrée est modélisée comme une charge répartie sur un disque (ou rectangle équivalent) de diamètre égal à l'épaisseur du dallage.

On a représenté sur la figure 30 la variation du rapport $\mathrm{Mx} / \mathrm{Q}$ au droit de l'axe $\mathrm{X}^{\prime} \mathrm{X}$, ce qui permet d'avoir un résultat indépendant de la valeur de la charge appliquée Q. L'amplitude du moment fournie par l'approche du DTU 13.3 est de $0,125 \mathrm{Q}$. Cette valeur est deux fois plus faible que celle obtenue par une approche plus élaborée. Le fait que dans le DTU, le calcul des sollicitations soit basé sur la courbure du support, peut expliquer en partie l'écart constaté : la figure 27 montre en effet que la courbure maximale du profil de tassement obtenu par l'approche du DTU est relativement sousestimée par rapport aux deux autres approches.

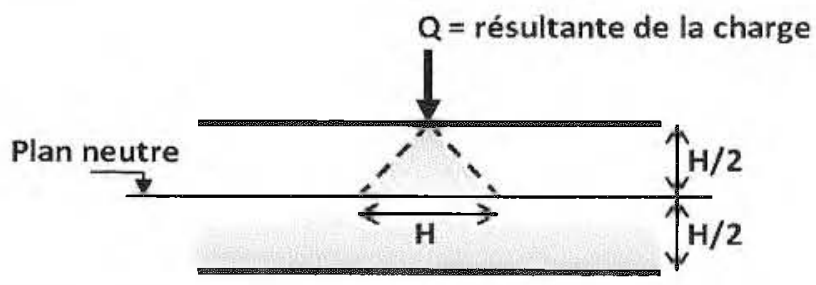

F1G. 29 Diffusion d'une charge concentrée. Diffusion of a point load.

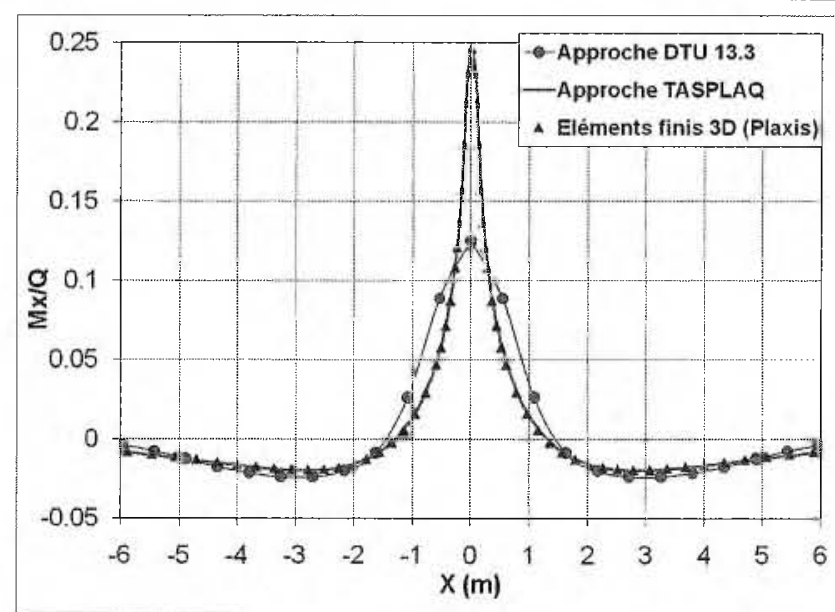

FIG. 30 Cas d'une charge isolée : moment fléchissant $\mathrm{Mx}$ (axe $\mathrm{X}^{\prime} \mathrm{X}$ ).

Case of a point load: bending moment $\mathrm{Mx}\left(\mathrm{X}^{\prime} \mathrm{X}\right.$ axis). 


\section{Cas de deux charges voisines}

On considère à présent le cas de deux charges de $500 \mathrm{kN}$ chacune, espacées de $4 \mathrm{~m}$ (Fig. 26). Sur la figure 31, on a représenté les tassements issus des trois approches. On constate que l'approche du DTU aboutit à une allure de tassement sensiblement différente : le tassement maximal est atteint entre les deux charges contrairement à ce qui est obtenu par un calcul plus complet. En effet, l'approche du DTU est basée sur le principe d'un disque d'interaction, associé à chaque charge, et sur lequel la réaction du sol est uniforme. Dans le cas présent, les deux disques d'interaction se superposent (diamètre équivalent égal à 5,42 m supérieur à la distance séparant les deux charges) et créent une zone de réaction cumulée plus forte au milieu, qui ne correspond pas toujours à la réalité (Fig. 32).
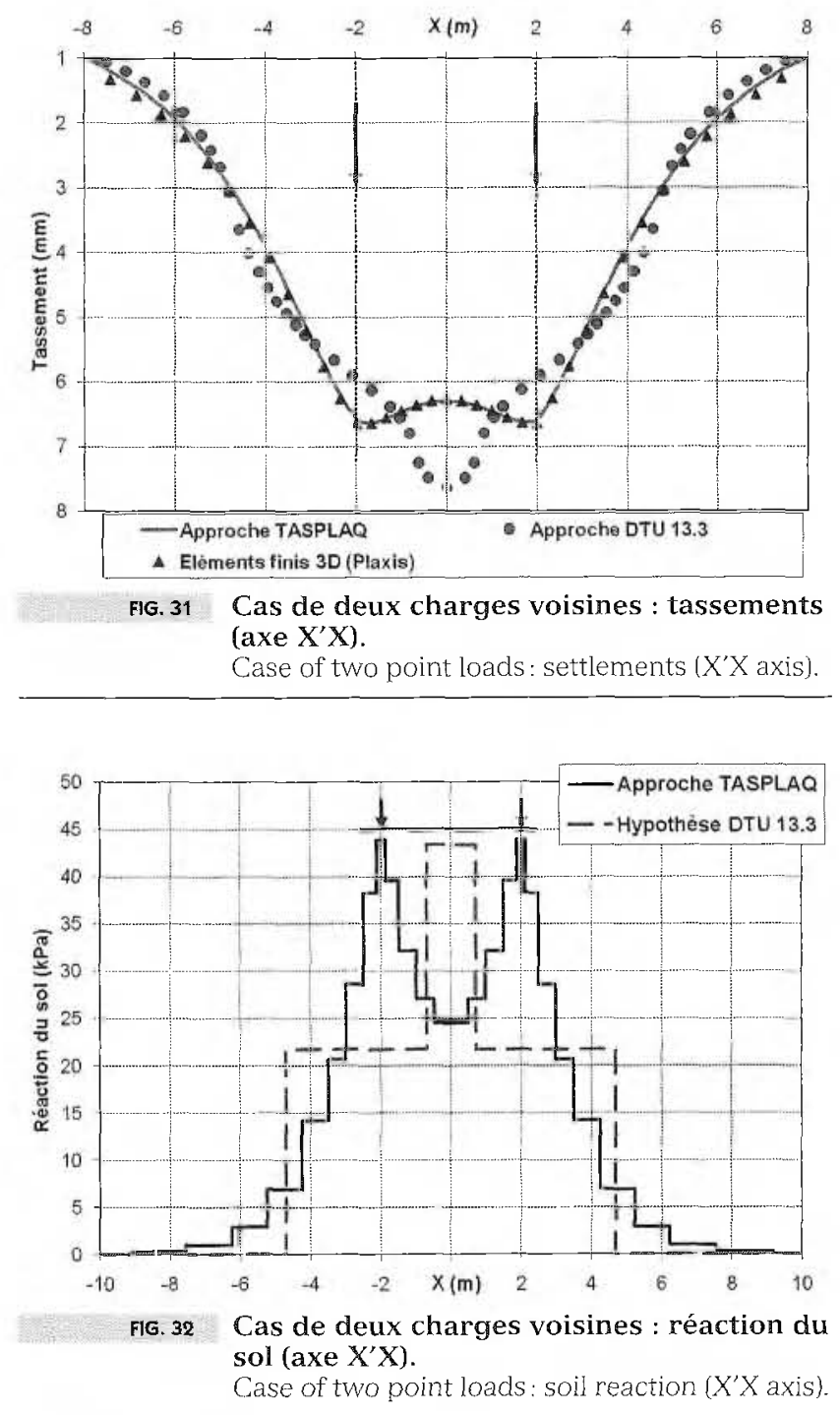

Ces exemples simples sont destinés à iliustrer les capacités de la méthode TASPLAQ à traiter les problèmes de dallage, mais également à mettre en lumière les simplifications inhérentes à l'approche simplifiée décrite dans le DTU 13.3, dont il convient que le projeteur soit convenablement averti. A ce propos il peut être rappelé que le DTU 13.3 précise que la méthode simplifiée est à utiliser à défaut de méthodes plus rigoureuses, éprouvées et scientifiquement justifiées. Si on s'intéresse aux déformations et sollicitations d'origine ( mécanique », alors le modèle TASPLAQ a précisément pour vocation de figurer parmi ces méthodes avancées.

\section{9}

\section{Extension de la méthode}

Dans le cas de dallages par exemple, l'existence d'une couche de forme généralement plus raide en surface peut paraître de nature à limiter la validité du modèle TASPLAQ, du fait de l'inadéquation des formules de Boussinesq dans ce cas précis. L'expérience acquise par les nombreux tests de validation montre néanmoins que, dans une telle situation, la rigidité propre à l'élément de structure (plaque du radier ou dallage) qui vient ( coiffer » cette couche peu déformable atténue notablement les écarts en tassement qui seraient obtenus sans cet élément de structure.

Une amélioration de l'approche de base qui a été décrite, permettant de mieux surmonter cette limitation potentielle, est possible par une technique d'homogénéisation. Celle-ci consiste à définir une inertie équivalente de l'ensemble \{dallage + couche de forme\}, puis à traiter le problème de l'appui de cette plaque “ homogénéisée » sur les couches sous-jacentes.

Cette fonctionnalité spécifique a également été introduite. L'inertie équivalente qui est définie peut être variable dans le modèle afin de tenir compte de la variation de l'épaisseur du milieu équivalent, notamment au droit des joints. Cette technique conduit effectivement à une déformée plus précise, et il est possible de remonter aux efforts dans la plaque à partir de ceux du milieu équivalent en effectuant une « déshomogénéisation u de celui-ci.

Il convient toutefois de s'assurer de la compatibilité des contraintes de flexion obtenues dans la couche de forme avec les contraintes initiales et/ou les propriétés rhéologiques du matériau qui la constitue.

\section{0}

\section{Conclusion}

La méthode développée pour l'étude de l'interaction sol-plaque n'est pas, bien entendu, aussi puissante et versatile que celle des éléments finis en trois dimensions. Mais elle présente des avantages décisifs en s'affranchissant du maillage du sol et en limitant l'emprise du modèle à celle des éléments de fondations.

Cette méthode a été basée sur un couplage entre une formulation en éléments finis de plaque et les formules de Boussinesq, et conduit, dans le domaine de sa validité, à des résultats voisins de ceux obtenus par traitement complet en éléments finis 3D. Elle s'applique à tout massif de fondation constitué de couches horizontales, dont le comportement peut être considéré élastique dans la situation de chargement étudiée. De la sorte cette méthode se substitue avantageusement aux méthodes de calcul de plaques sur appuis élastiques en évitant la simplification très critiquée de la proportionnalité réaction-déplacement, et par conséquent l'écueil du choix d'un module de réaction dont chacun sait qu'il n'est caractéristique ni d'un sol ni d'un ensemble sol-structure. 
Cette méthode a fait l'objet d'un programme informatique, qui permet de traiter le tassement et la déformée de plaques d'inertie variable, sous chargement ponctuel et/ou réparti. Le mudèle a été perfectionné par l'introduction de plusieurs fonctionnalités comme la prise en compte d'une plaque de géométrie quelconque, la modélisation de plusieurs plaques désolidarisées ainsi que la gestion automatique du décollement de la plaque. Il est également possible de prendre en compte, de manière approchée, tout seuil de plastification prédéfini par l'utilisateur en sous-face de la plaque ou l'influence de l'état initial des contraintes pour traiter d'une fondation placée au fond d'une excavation.

Une extension du domaine d'application via une technique d'homogénéisation est également rendue possible et s'avère intéressante dans le cas particulier d'un massif de fondation comportant une couche de forme raide en surface.

\section{Bibliographie}

Batoz J.-L., Dhatt G. - Modélisation des structures en éléments finis. Presses de I'université Laval, édition Hermès, 1990. Burland J.B., Broms B.B., De Mello V.F.B. - Behaviour of Foundations and Structures. Proceedings of the ninth international conterence on soil mechanics and foundation engineering. Tokyo, vol. 2 , 1977, p. 495-546.

Cassan M., Guendon J.-P., Locci J.-M. Méthode numérique pour l'étude de l'interaction sul-radier. 10: Conférence européenne de mécanique des sols et des travaux de fondations, Florence (Italie), vol. 1, 26-30 mai 1991,p. 363-366.

Combarieu O. - Ĺusage des modules de déformation en géotechnique. Revue française de géotechnique, $\mathrm{n}^{\circ} 114,1^{\mathrm{er}}$ trimestre 2006.

Norme NF.P.11-213, DTU 13.3 - Dallages: conception, calcul et exécution. Partie 1 cahiers des clauses techniques des dallages à usage industriel ou assimilés.

Pliskin L. - Dallages industriels sur sol. Revue française de génie civil, vol. 3 , $n^{\circ}$ 6, 1999, p. 423-436.
Potts D., Zdravkovic L. - Finite element analysis in geotechnical engineering. Thomas Telford Publishing, 1999.

Terzaghi K. - Theoretical soil mechanics - Theory of Semi-infinite elastic solids 1943, $2^{\text {nu }}$ edition.

Vezole P. - Interactions sol structure. Méthode de calcul des pressions normales à l'interface dans quelques cas courants. Annales de l'TTBTP, $\mathrm{n}^{\circ} 448$, octobre 1986.

Zienkiewicz O.C., Taylor R.L. - The finite Element Method. McGraw-Hill book Company (UK), 1991, $4^{\text {th }}$ edition. 